\title{
MUTUAL INDUCTANCE AND FORCE BETWEEN TWO COAXIAL HELICAL WIRES
}

\author{
By Chester Snow
}

\section{ABSTRACT}

A formula is found for the mutual inductance and force between two coaxial helical wires which, in addition to the well-known current-sheet formula, contains small correction terms, one of which represents the axial components of current; one the finite diameters of the wires; and another $\omega_{a}$, which depends upon the relative azimuths of the helices, arises, naturally, from the actual helical form of the windings. The pitch of the windings may be different in the two, but each is considered so small in comparison with the cylindrical radii that terms relatively smaller than the square of this ratio may be neglected. The number of turns is not necessarily an integer.

\section{CONTENTS}

Page

I. Introduction

II. Formal expressions for the mutual inductance and force between two coaxial helices........ 241

III. The principal terms $\omega_{\theta}$ and $\omega_{\theta}^{\prime} \ldots \ldots$

IV. Effect of axial current in helices and lead wires...

V. The azimuthal terms

VI. The helix equivalent to a helical wire

VII. Application to the current balance used in the National Physical Laboratory _... 266

VIII. Summary

\section{INTRODUCTION}

A formula of precision sufficient for absolute electrical measurements of the mutual inductance or force between two coaxial helical wires does not appear to have been developed to the same degree of precision as in the case of a self inductance. The principal part corresponding to current sheets is well known, but even if the construction were perfect, there remain certain small correction terms which must be found by starting with an idealization of the coils which is nearer the actual than a current sheet. The procedure here adopted as the most natural is to formulate the mutual inductance as Neumann's doubleline integral and to expand the integrand in a Fourier's series as a function of the difference of the angular parameters of the two helices. The constant term of this series gives the current sheet formula, provided both angular and axial components of current are included in this term. The remainder of the series gives a correction depending upon azimuth of the two helices, which is relatively small for such closely wound coils as are used in practice. By restricting the problem to cases where the windings of the one do not come too close to those 
of the other, we avoid most of those evaluations of proximity effect so troublesome in deriving a formula for self inductance.

The equations defining the first helix, $h_{1}$, may be taken as the three equations which express the rectangular coordinates, $x_{1}, y_{1}, z_{1}$, of any point, $P_{1}$, on it, in terms of a single independent parameter. The most suitable line parameter in this case is the angle $\theta_{1}$, where $x_{1}, r_{1}$, and $\theta_{1}$ are the cylindrical coordinates of $P_{1}$. If the rectangular axes are right-handed, a positive pitch, $2 \pi p_{1}$, corresponds to a right-handed helix. If the plane $x=x_{i_{1}}$ and the azimuth $\theta=\theta_{i_{1}}$ are those of its initial point, and $x=x_{e_{1}}, \theta=\theta_{e_{1}}$ its end point, and if $r_{1}$ is its cylindrical radius, the equations of $h_{1}$ are

$$
\left.\begin{array}{l}
x_{1}=x_{i_{1}}+p_{1}\left(\theta_{1}-\theta_{i_{1}}\right) \\
y_{1}=r_{1} \cos \theta_{1} \\
z_{1}=r_{1} \sin \theta_{1}
\end{array}\right\} \text {, where } \theta_{i_{1}} \leqq \theta_{1} \leqq \theta_{e_{1}} .
$$

Its axial length, $l_{1}$, is given by

$$
l_{1}=x_{e_{1}}-x_{i_{1}}=p_{1}\left(\theta_{e_{1}}-\theta_{i_{1}}\right) \equiv 2 \pi p_{1} N_{1} .
$$

Its element of length has the magnitude

$$
d s_{1}=\sqrt{r_{1}^{2}+p_{1}^{2}} d \theta_{1}
$$

and the direction cosines

$$
\frac{d x_{1}}{d s_{1}}=\frac{p_{1}}{\sqrt{r_{1}^{2}+p_{1}^{2}}}, \frac{d y_{1}}{d s_{1}}=-\frac{r_{1} \sin \theta_{1}}{\sqrt{r_{1}^{2}+p_{1}^{2}}}, \frac{d z_{1}}{d s_{1}}=\frac{r_{1} \cos \theta_{1}}{\sqrt{r_{1}^{2}+p_{1}^{2}}} .
$$

If two helices of the type in eq 1 differ only in the values of their two constants, $x_{i_{1}}$ and $r_{1}$, they may be considered as filaments of the same helical wire, $w_{1}$, where, for the purpose of this paper, a "helical wire" is defined as follows:

To specify a helical wire, $w_{1}$, of pitch $2 \pi p_{1}$ and axial length $l_{1}$ and with a wire radius, $\rho_{1}$, whose central filament has the cylindrical radius $r_{1}$, the initial plane and azimuth of this central filament being $x_{i_{1}}$ and $\theta_{i_{1}}$, the plane and azimuth of its end point, $x_{e_{1}}$ and $\theta_{e_{1}}$, we define it as the totality of all helices, $h_{1}{ }^{\prime}$, represented by eq 1 with $\left(x_{i_{1}}{ }^{\prime}\right.$ and $r_{1}{ }^{\prime}$ in place of $\left.x_{i_{1}}, r_{1}\right)$, provided that the two constants, $x_{i_{1}}{ }^{\prime}$ and $r_{1}{ }^{\prime}$, lie in the range

$$
\left(x_{i_{1}}{ }^{\prime}-x_{i_{1}}\right)^{2}+\left(r_{1}{ }^{\prime}-r_{1}\right)^{2} \leqq \rho_{1}{ }^{2}
$$

All azimuthal planes cut this wire in circular sections of radius $\rho_{1}$, the initial face of the wire in the plane $\theta=\theta_{i_{1}}$, and its end face in the plane $\theta=\theta_{e_{1}}$. All its filaments have the same terminal azimuths and the same axial length $l_{1}$. Giving $\theta_{i_{1}}$ a variation while holding $l_{1}$ constant corresponds to a rigid rotation of the wire about the $x$ axis, while a variation in $x_{i 1}$ with $l_{1}$ constant is a translation parallel to that axis.

The second helical wire, $w_{2}$, coaxial with $w_{1}$ is specified, as above, with subscripts 2 . It is assumed that $p_{1}$ and $p_{2}$ are both positive; that is, $w_{1}$ and $w_{2}$ are both right-handed helical axes or both lefthanded, depending upon whether the coordinate axes are chosen right-handed or left-handed. 
The total "number of turns," $N_{1}$ and $N_{2}$, are not necessarily integers, as no essential simplification in the final formulas would be obtained by placing such a restriction upon the generality of their application.

The associated current sheet of a helix is defined as a circular cylindrical surface coaxial with it, having the same radius and end planes, on which the linear density of current has the angular component $j_{\theta}=n=1 / 2 \pi p$ and the axial component $j_{x}=1 / 2 \pi r$. The mutual inductance between the sheets of the two helices will be denoted by $m_{v_{1} s_{2}}=m_{\theta}+m_{x}$.

The mutual inductance between one helix and the current sheet of the other is identical with that of the two sheets, and this statement holds for each component of $m_{s_{1} s_{2}}$ separately, the part $m_{\theta}$ being due to their angular components of current and $m_{x}$ due to their axial currents. The latter, $m_{x}$, may also be interpreted as the mutual inductance between one of the sheets and any straight line which is a generator of the other.

A definition will be given later of a helix which is equivalent to a helical wire, so that the associated current sheet of a helical wire is that of its equivalent helix.

The equivalent helix of a wire has the same pitch, terminal end planes, (and hence axial length), as the central filament of the wire, but a slightly different cylindrical radius, depending upon the wire radius and the nature of the current distribution in the wire, which is assumed to flow everywhere in the direction of the generating helical filament, its magnitude being a function of $r$.

Hence the axial length adopted here for that of the current sheet of a helical wire is in harmony with that generally accepted. All single-layer solenoids are, in fact, helical wires, but when $N$ is an integer they have generally been treated by idealizing them as $N$ equal coaxial circular turns of wire, their central planes equally spaced at $x=2 \pi p n, n=0,1,2,3, \ldots N-1$, so that the distance between central planes of the first and last turn is $2 \pi p N-2 \pi p$. If all these circular turns are cut by a plane through the $x$ axis and each given a shear, they go exactly into the "helical wire" here defined. The current sheet associated with this series of circular turns of wire is generally taken with length $2 \pi p$ plus the axial distance $2 \pi p N-2 \pi p$ between central planes of the first and last wire, that is, $2 \pi p N$, which is the length adopted here in general, although we do not restrict $N$ to be integral.

\section{FORMAL EXPRESSIONS FOR THE MUTUAL INDUCT- ANCE AND FORCE BETWEEN TWO COAXIAL HELICES}

The mutual inductance, $m$, between the helix $h_{1}$, and that $h_{2}$ is here defined by the Neumann's double-line integral

$$
m=\int d s_{1} \int d s_{2} \frac{\cos \left(d s_{1}, d s_{2}\right)}{\sqrt{\left(x_{2}-x_{1}\right)^{2}+\left(y_{2}-y_{1}\right)^{2}+\left(z_{2}-z_{1}\right)^{2}}} \cdot
$$

Letting

$$
R^{2}(\psi)=r_{1}{ }^{2}+r_{2}{ }^{2}-2 r_{1} r_{2} \cos \psi,
$$


this becomes

$$
m=\int_{x_{i_{1}}}^{x_{o_{1}}} d x_{1}^{x_{e_{2}}} d x_{i_{i_{2}}} \frac{\frac{r_{1} r_{2}}{p_{1} p_{2}} \cos \psi\left(x_{1}, x_{2}\right)+1}{\sqrt{\left(x_{2}-x_{1}\right)^{2}+R^{2}\left[\psi\left(x_{1}, x_{2}\right)\right]}}
$$

where $\psi\left(x_{1}, x_{2}\right)$ is a function of $\left(x_{1}, x_{2}\right)$ given by

$$
\psi\left(x_{1}, x_{2}\right)=\frac{x_{2}}{p_{2}}+\left[\theta_{i_{2}}-\frac{x_{i_{2}}}{p_{2}}\right]-\frac{x_{1}}{p_{1}}-\left[\theta_{i_{1}}-\frac{x_{i_{1}}}{p_{1}}\right]
$$

The part of $m$ which is due to the angular components of current in the two helices is that part of the integral in eq 7 attributable to the first part of the numerator $r_{1} r_{2} \cos \psi / p_{1} p_{2}$; the part coming from the item 1 in the numerator is due to their $x$-components or axial components of current. The latter is a small quantity compared with the former (in general, of second order, when $p_{1} / r_{1}$ and $p_{2} / r_{2}$ are both small quantities of the first order).

Writing eq 7 in the form

$$
m=\int_{x_{i_{1}}}^{x_{i_{1}}} d x_{1} \int_{x_{i_{2}}}^{x_{i_{2}}} d x_{2} f\left[x_{2}-x_{1}, \psi\left(x_{1}, x_{2}\right)\right]
$$

and changing the variable $x_{2}$ to $x^{\prime}$ by the substitution $x^{\prime}=x_{2}-x_{1}$ gives

$$
m=\int_{x_{i_{1}}}^{x_{i_{1}}} d x_{1} \int_{x_{i_{2}}-x_{1}}^{x_{e_{2}}-x_{1}} f\left[x^{\prime}, \psi\left(x_{1}, x^{\prime}+x_{1}\right)\right] d x^{\prime}
$$

Next, changing the variable $x_{1}$ to $x^{\prime \prime}$ by the substitution $x^{\prime \prime}=x_{e 2}-x_{1}$ gives

$$
\begin{aligned}
& m=\int_{x_{e_{2}}-x_{e_{1}}}^{x_{e_{2}}-x_{i_{1}}} d x^{\prime \prime} \int_{x^{\prime \prime}-\left(x_{e_{2}}-x_{i_{2}}\right)}^{x^{\prime \prime}} f\left[x^{\prime}, \psi\left(x_{e 2}-x^{\prime \prime}, x^{\prime}-x^{\prime \prime}+x_{e 2}\right)\right] d x^{\prime} \\
& =\int_{x_{e_{2}}-x_{e_{1}}}^{x_{e_{2}}-x_{i_{1}}} d x^{\prime \prime}\left\{\int_{0}^{x^{\prime \prime}} f\left[x^{\prime}, \psi\left(x_{e 2}-x^{\prime \prime}, x_{e 2}-x^{\prime \prime}+x^{\prime}\right)\right] d x^{\prime}\right. \\
& \left.\quad-\int_{0}^{x^{\prime \prime}-\left(x_{e_{2}}-x_{i_{2}}\right)} f\left[x^{\prime}, \psi\left(x_{e 2}-x^{\prime \prime}, x_{e 2}-x^{\prime \prime}+x^{\prime}\right)\right] d x^{\prime}\right\} \\
& =\int_{0}^{x_{e_{2}}-x_{i_{1}}} d x^{\prime \prime} \int_{0}^{x^{\prime \prime}} f\left[x^{\prime}, \psi\left(x_{e 2}-x^{\prime \prime}, x_{e 2}-x^{\prime \prime}+x^{\prime}\right] d x^{\prime}\right. \\
& -\int_{x_{e_{2}}-x_{e_{1}}}^{x_{e_{2}}-x_{i_{1}}} d y \int_{0}^{\prime \prime} \int_{0}^{x^{\prime \prime}} f\left[x^{\prime}, \psi\left(x_{e 2}-x^{\prime \prime}, x_{e 2}-x^{\prime \prime}+x^{\prime}\right] d x^{\prime}\right.
\end{aligned}
$$

In the last double integral, the variable of integration $x^{\prime \prime}$ has merely been replaced by $y$ in order that the symbol $x^{\prime \prime}$ may be used in another sense, so that the four integrals into which $m$ is resolved in eq 9 below shall each have the same designation of their two variables, and shall have limits of the same general form. In the last double integral we next change the variable $y$ to $x^{\prime \prime}$ by the substitution $x^{\prime \prime}=y-\left(x_{e q}-x_{i_{2}}\right)$, 
and it becomes $+\int_{x_{i_{2}}-x_{i_{1}}}^{x_{i_{1}}-x_{i_{1}}} d x^{\prime \prime} \int_{0}^{x^{\prime \prime}} f\left[x^{\prime}, \psi\left(x_{i_{2}}-x^{\prime \prime}, x_{i_{2}}-x^{\prime \prime}+x^{\prime}\right)\right] d x^{\prime}$.

Writing the $x^{\prime \prime}$ integral of this in the form

gives

$$
\int_{0}^{x_{i_{2}}-x_{\ell_{1}}}() d x^{\prime \prime}-\int_{0}^{x_{i_{2}}-x_{i_{1}}}() d x^{\prime \prime}
$$

$$
\begin{aligned}
m & =\int_{0}^{x_{c_{2}}-x_{i}} d x^{\prime \prime} \int_{0}^{x^{\prime}} f\left[x^{\prime}, \psi_{e_{3} i_{1}}\left(x^{\prime}, x^{\prime \prime}\right)\right] d x^{\prime} \\
& -\int_{0}^{x_{c_{2}}-x_{x_{1}}} d x^{\prime \prime} \int_{0}^{x^{\prime}} f\left[x^{\prime}, \psi_{e_{2} e_{1}}\left(x^{\prime}, x^{\prime \prime}\right)\right] d x^{\prime} \\
& +\int_{0}^{x_{i_{2}}-x_{i_{1}}} d x^{\prime \prime} \int_{0}^{x^{\prime}} f\left[x^{\prime}, \psi_{i_{2} e_{1}}\left(x^{\prime}, x^{\prime \prime}\right)\right] d x^{\prime} \\
& -\int_{0}^{x_{i_{2}}-x_{i_{1}}} d x^{\prime \prime} \int_{0}^{x^{\prime}} f\left[x^{\prime}, \psi_{i_{i_{1}} i_{1}}\left(x^{\prime}, x^{\prime \prime}\right)\right] d x^{\prime},
\end{aligned}
$$

where

$$
\left.\begin{array}{l}
\psi_{e_{2} i_{1}}\left(x^{\prime}, x^{\prime \prime}\right)=\frac{x^{\prime}}{p_{2}}+\left(\frac{1}{p_{1}}-\frac{1}{p_{2}}\right) x^{\prime \prime}+\theta_{e_{2}}-\theta_{i_{1}}-\left(\frac{x_{e_{2}}-x_{i_{1}}}{p_{1}}\right) \\
\psi_{e_{e_{1}}\left(x^{\prime}\right.}\left(x^{\prime \prime}\right)=\frac{x^{\prime}}{p_{2}}+\left(\frac{1}{p_{1}}-\frac{1}{p_{2}}\right) x^{\prime \prime}+\theta_{e_{2}}-\theta_{e_{1}}-\left(\frac{x_{e_{2}}-x_{e_{1}}}{p_{1}}\right) \\
\psi_{i_{2} e_{1}}\left(x^{\prime}, x^{\prime \prime}\right)=\frac{x^{\prime}}{p_{2}}+\left(\frac{1}{p_{1}}-\frac{1}{p_{2}}\right) x^{\prime \prime}+\theta_{i_{2}}-\theta_{e_{1}}-\left(\frac{x_{i_{2}}-x_{e_{1}}}{p_{1}}\right) \\
\psi_{i_{2} i_{1}}\left(x^{\prime}, x^{\prime \prime}\right)=\frac{x^{\prime}}{p_{2}}+\left(\frac{1}{p_{1}}-\frac{1}{p_{2}}\right) x^{\prime \prime}+\theta_{i_{2}}-\theta_{i_{1}}-\left(\frac{x_{i_{2}}-x_{i_{1}}}{p_{1}}\right)
\end{array}\right\}
$$

In what follows we shall use $x$ (positive or negative) to represent one of the following four $x$-differences, and $\theta_{x}$ for the associated constant azimuth-difference.

$$
\left.\begin{array}{c}
x=x_{e_{2}}-x_{i_{1}}, x_{e_{3}}-x_{e_{1}}, x_{i_{3}}-x_{e_{1}}, \text { and } x_{i_{3}}-x_{i_{1}} \\
\theta_{x}=\theta_{e_{2}}-\theta_{i_{1}}, \theta_{e_{2}}-\theta_{e_{1}}, \theta_{i_{2}}-\theta_{e_{1}} \text {, and } \theta_{i_{3}}-\theta_{i_{1}}
\end{array}\right\}
$$

These four differences represent the four possible axial distances between a terminal plane of $h_{1}$ and a terminal plane of $h_{2}$. The corresponding azimuth differences, $\theta_{x}$, are those of the respective pairs of terminals (having the same subscripts). Equation 10 may by a similar notation be condensed into

$$
\psi_{x}\left(x_{1}, x_{2}\right)=\frac{x_{1}}{p_{2}}+\left(\frac{1}{p_{1}}-\frac{1}{p_{2}}\right) x_{2}+\theta_{x}-\frac{x}{p_{1}},
$$

where $\psi_{x}=\psi_{e_{2} i_{1}}, \psi_{e_{2} e_{1}}, \psi_{i_{2} e_{1}}$, and $\psi_{i_{2} i_{1}}$.

From the definitions given in eq 11 and 12 it is found by the use of eq 1 and 2 that $\psi_{e_{3} i_{1}}=\psi_{e_{2} e_{1}}$ and $\psi_{i_{2} e_{1}}=\psi_{i_{2} t_{1}}$. 
Hence eq 9 shows that the mutual inductance between two helices is of the form

$$
m=\omega\left(x_{e_{2}}-x_{i_{1}}\right)-\omega\left(x_{e_{2}}-x_{e_{1}}\right)+\omega\left(x_{i_{2}}-x_{e_{1}}\right)-\omega\left(x_{i_{2}}-x_{i_{1}}\right)
$$

Consequently, the $x$-component, $f$, of the attraction of $h_{2}$ for $h_{1}$, when each carries unit current in the same direction, is given by

$$
f=-\omega^{\prime}\left(x_{e_{2}}-x_{i_{1}}\right)+\omega^{\prime}\left(x_{e_{2}}-x_{e_{1}}\right)-\omega^{\prime}\left(x_{i_{2}}-x_{e_{1}}\right)+\omega^{\prime}\left(x_{i_{2}}-x_{i_{1}}\right),
$$

where primes denote derivatives with respect to the $x$-argument and

$$
\omega(x)=\int_{0}^{x} d x_{2} \int_{0}^{x_{2}} \frac{\frac{p_{1} p_{2}}{p_{1} p_{2}} \cos \psi_{x}+1}{\sqrt{x_{1}^{2}+R^{2}\left(\psi_{x}\right)}} d x_{1},
$$

where $R$ is a function of $\psi_{x}$ defined by eq 6 and $\psi_{x}$ is defined by eq 12 . It will be found that $\omega$ is an even function and $\omega^{\prime}$ an odd function of the $x$-argument, both vanishing with it.

It is necessary to evalute the integral $\omega(x)$ for positive and negative values of $x$ in order to treat all possible coaxial helices. To do this we note that the integrand in the integral of eq 14 is an even periodic function of $\psi$, with period $2 \pi$, and may therefore be developed in a Fourier's cosine series. To find this series, consider the function $k / 4 \sqrt{1-k^{2} \cos ^{2} \theta}$, where the modulus, $k$, is a positive real in the range $0 \leqq k \leqq 1$. This function has the development

$$
\frac{k}{4 \sqrt{1-k^{2} \cos ^{2} \theta}}=\frac{1}{2} \phi_{0}(k)+\sum_{n=1}^{\infty} \phi_{n}(k) \cos 2 n \theta,
$$

where the functions $\phi_{n}(k)$ are given by

$\phi_{n}(k)=\phi_{-n}(k)=\frac{k}{\pi} \int_{0}^{\frac{\pi}{2}} \frac{\cos 2 n \theta}{\sqrt{1-k^{2} \cos ^{2} \theta}} d \theta=\frac{(-1)^{n} k}{\pi} \int_{0}^{\frac{\pi}{2}} \frac{\cos 2 n \theta}{\sqrt{1-k^{2} \sin ^{2} \theta}} d \theta$.

They may be expressed in terms of the hypergeometric series

$$
\phi_{n}(k)=\frac{k^{2 n+1}}{2 \pi} \frac{\Gamma\left(n+\frac{1}{2}\right) \Gamma\left(n+\frac{1}{2}\right)}{\Gamma(2 n+1)} F\left(n+\frac{1}{2}, n+\frac{1}{2}, 2 n+1, k^{2}\right) .
$$

When $k \rightarrow 1$ every $\phi_{n} \rightarrow \infty$, its principal part being $\phi_{n} \cong \frac{1}{\pi} \cdot \log \frac{1}{k^{\prime}}$, where $k^{\prime}$ is the complementary modulus. These functions satisfy the differential equation

$$
k^{3} \frac{d}{d k}\left[\frac{1-k^{2}}{k} \phi_{n}^{\prime}(k)\right]=\left(4 n^{2}-1\right) \phi_{n}(k),
$$

where $\phi_{n}{ }^{\prime}=\frac{d}{d k} \phi_{n}$.

The first two functions of the series, $\phi_{0}$ and $\phi_{1}$, are expressible in terms of the two complete elliptic integrals of the first and second kind with modulus $k$. 


$$
\phi_{0}(k)=\frac{k}{\pi} K(k) \text { and } \phi_{1}(k)=\frac{2}{\pi}\left(\frac{K(k)-E(k)}{k}\right)-\frac{k K(k)}{\pi}
$$

Any other function, $\phi_{n}(k)$, could be computed in terms of $\phi_{0}$, and $\phi_{1}$ by successive application of the recurrence relation

$$
\left(n+\frac{1}{2}\right) \phi_{n+1}+\left(n-\frac{1}{2}\right) \phi_{n-1}=2 n\left(\frac{2}{k^{2}}-1\right) \phi_{n}
$$

The derivative, $\phi_{n}^{\prime}$ is given by

$$
\frac{1-k^{2}}{k} \phi_{n}^{\prime}(k)=\left(n-\frac{1}{2}\right)\left[\phi_{n-1}-\left(\frac{2}{k^{2}}-1\right) \phi_{n}\right]
$$

When $k$ is a function of $x, r_{1}$, and $r_{2}$ defined by

$$
k^{2}=\frac{4 r_{1} r_{2}}{x^{2}+\left(r_{1}+r_{2}\right)^{2}} .
$$

the mutual inductance of two coaxial circles of radii $r_{1}$ and $r_{2}$ (the distance between their planes being $x$ ) is $M=4 \pi^{2} \sqrt{r_{1} r_{2}} \phi_{1}(k)$

Their attraction, $F$, with unit currents is $F=-D_{x} M$, so that

$$
Y_{1}(k) \equiv 4 \pi^{2} \phi_{1}(k)=\frac{M}{\sqrt{r_{1} r_{2}}} \text { and } Y_{2}(k) \equiv \pi^{2} k^{3} \phi_{1}^{\prime}(k)=\frac{\sqrt{r_{1} r_{2}}}{x} F
$$

These are convenient for finding $\phi_{1}(k)$ and $\phi_{1}^{\prime}(k)$, because the functions $Y_{1}(k)$ and $Y_{2}(k)$ are tabulated against $k^{2}$ in table 2 of the Scientific Papers of the Institute of Physical and Chemical Research (Komagome, Hongo, Tokyo, 1927) by Nagaoka and Sakurai.

When $k$ is given by eq 22 , the functions $\phi_{n}(k)$ satisfy the partial differential equation

$$
\begin{aligned}
& \left\{D_{x}^{2}+D_{r_{1}}^{2}+\frac{1-2 \alpha}{r_{1}} D_{r_{1}}+\frac{\alpha^{2}-n^{2}}{r_{1}^{2}}\right\}\left(r_{1}^{\alpha-\frac{1}{2}} \phi_{n}\right)=0 \\
& =\left\{D_{x}^{2}+D_{r_{2}}^{2}+\frac{1-2 \alpha}{r_{2}} D_{r_{2}}+\frac{\alpha^{2}-n^{2}}{r_{2}^{2}}\right\}\left(r_{2}{ }^{a-\frac{1}{2}} \phi_{n}\right),
\end{aligned}
$$

where $\alpha$ is any constant.

There are also the integral representations

$$
\frac{\phi_{n}(k)}{\sqrt{r_{1} r_{2}}}=\frac{1}{2 \pi} \int_{0}^{2 \pi} \frac{\cos n \psi}{\sqrt{x^{2}+R^{2}(\psi)}} d \psi=\int_{0}^{\infty} e^{-s|x|} J_{n}\left(r_{1} s\right) J_{n}\left(r_{2} s\right) d s,
$$

where $J_{n}$ is Bessel's function. The modulus, $k$, in all that follows, will be understood as the function of $x, r_{1}$, and $r_{2}$ defined by eq 22 , unless otherwise stated. When $x$ is replaced by $x_{1}$ it will be called $k_{1}$, and $k_{0}$ means the modulus for $x=0$.

Hence, as shown by eq 15 ,

$$
\frac{1}{\sqrt{x_{1}^{2}+R^{2}\left(\psi_{x}\right)}}=\frac{1}{\sqrt{r_{1} r_{2}}}\left\{\phi_{0}\left(k_{1}\right)+2 \sum_{n=1}^{\infty} \phi_{n}\left(k_{1}\right) \cos n \psi_{x}\right\},
$$


so that the required Fourier's series for the integrand of eq 14 is

$$
\begin{aligned}
\frac{\frac{r_{1} r_{2}}{p_{1} p_{2}} \cos \psi_{x}+1}{\sqrt{x_{1}^{2}+R_{1}^{2}\left(\psi_{\tau}\right)}}= & \frac{1}{\sqrt{r_{1} r_{2}}}\left\{\frac{r_{1} r_{2}}{p_{1} p_{2}} \phi_{1}\left(k_{1}\right)+\phi_{0}\left(k_{1}\right)\right. \\
& \left.+\sum_{n=1}^{\infty} \cos n \psi_{x}\left[\frac{r_{1} r_{2}}{p_{1} p_{2}}\left(\phi_{n-1}\left(k_{1}\right)+\phi_{n+1}\left(k_{1}\right)\right)+2 \phi_{n}\left(k_{1}\right)\right]\right\}
\end{aligned}
$$

Hence eq 14 may be put in the form

where

$$
\omega(x)=\omega_{\theta}(x)+\omega_{x}(x)+\omega_{a}\left(x, \theta_{x}\right),
$$

$$
\begin{aligned}
\omega_{s 182}=\omega_{\theta}+\omega_{x}= & \frac{\sqrt{r_{1} r_{2}}}{p_{1} p_{2}} \int_{0}^{x} d x_{2} \int_{0}^{x_{2}}\left[\phi_{1}\left(k_{1}\right)+\frac{p_{1} p_{2}}{r_{1} r_{2}} \phi_{0}\left(k_{1}\right)\right] d x_{1} \\
& =\frac{\sqrt{r_{1} r_{2}}}{p_{1} p_{2}} \int_{0}^{x}\left(x-x_{1}\right)\left[\phi_{1}\left(k_{1}\right)+\frac{p_{1} p_{2}}{r_{1} r_{2}} \phi_{0}\left(k_{1}\right)\right] d x_{1} \\
\omega_{a}\left(x, \theta_{x}\right) & =\sum_{n=1}^{\infty} \omega_{n}\left(x, \theta_{x}\right) \\
\omega_{x}\left(x, \theta_{x}\right) & =\frac{1}{\sqrt{r_{1} r_{2}}} \int_{0}^{x} d x_{2} \int_{0}^{x_{2}} d x_{1} \cos n \psi_{x}\left(x_{1}, x_{2}\right) \\
& {\left[\frac{r_{1} r_{2}}{p_{1} p_{2}}\left(\phi_{m-1}\left(k_{1}\right)+\phi_{n+1}\left(k_{1}\right)\right)+2 \phi_{\boldsymbol{k}}\left(k_{1}\right)\right] }
\end{aligned}
$$

The term $\omega_{\theta}$ is the part of the integral of eq 29 involving $\phi_{1}$; the term $\omega_{x}$ the small part involving $\phi_{0}$.

To prove that the $\omega_{31 s 2}$ defined by the integral of eq 29 is the $\omega$ function which, if used in the general formula, eq 13, would give the $m_{s 182}$ previously defined as mutual inductance of the two associated current sheets, we may start with the elementary formulation which is

$$
\begin{aligned}
m_{s 182} & =\int_{x_{i_{1}}}^{x_{x_{1}}} d x_{1} \int_{x_{i_{3}}}^{x_{x_{3}}} d x_{2} \int_{0}^{2 \pi} \frac{d \theta_{1}}{2 \pi} \int_{0}^{2 \pi} \frac{d \theta_{2}}{2 \pi} \frac{\frac{r_{1} r_{2}}{p_{1} p_{2}} \cos \left(\theta_{2}-\theta_{1}\right)+1}{\sqrt{\left(x_{2}-x_{1}\right)^{2}+R^{2}\left(\theta_{2}-\theta_{1}\right)}} \\
& =\int_{x_{i_{1}}}^{x_{x_{1}}} d x_{1} \int_{x_{i_{2}}}^{x_{\iota_{3}}} d x_{2} \int_{0}^{2 \pi} \frac{d \psi}{2 \pi} \frac{\frac{r_{1} r_{2}}{p_{1} p_{2}} \cos \psi+1}{\sqrt{\left(x_{2}-x_{1}\right)^{2}+R^{2}(\psi)}}
\end{aligned}
$$

By transformation similar to those used in passing from eq 7 to eq 13 (but much simpler) one finds that

$$
\begin{aligned}
& \int_{x_{i_{1}}}^{x_{i_{1}}} d x_{1} \int_{x_{i_{2}}}^{x_{i_{3}}} d x_{2} f\left(x_{2}-x_{1}\right)=\omega\left(x_{e 2}-x_{i 1}\right) \\
& -\omega\left(x_{e 2}-x_{e 1}\right)+\omega\left(x_{i 2}-x_{e 1}\right)-\omega\left(x_{i 2}-x_{i 1}\right)
\end{aligned}
$$


where

$\omega(x)=\int_{0}^{x} d x_{2} \int_{0}^{x_{2}} f\left(x_{1}\right) d x_{1}=\int_{0}^{x}\left(x-x_{1}\right) f\left(x_{1}\right) d x_{1}=\int_{0}^{-x} d x_{2} \int_{0}^{x_{2}} f\left(-x_{1}\right) d x_{1}$,

provided $f$ is such a function that these integrals converge. This shows that $\omega(x)$ is an even function of $x$, an odd function, or neither, according as $f(x)$ is. Also $\omega(0)=0$, and $\omega^{\prime}(0)=0$, where $\omega^{\prime}(x)=$ $D_{x} \omega(x)$. In eq $31 f(x)$ is an even function of $x$, so $\omega(x)$ is an even function of $x$, and $m_{s s}$ is therefore reduced to the general form of eq 13 , where the $\omega$-function is

$$
\begin{aligned}
\omega_{s_{1} s_{1}}=\omega_{\theta}+\omega_{x} & =\int_{0}^{x} d x_{2} \int_{0}^{x_{2}} d x_{1} \int_{0}^{2 \pi} \frac{d \psi}{2 \pi} \frac{\frac{r_{1} r_{2}}{p_{1} p_{2}} \cos \psi+1}{\sqrt{x_{1}^{2}+R_{i}^{2}(\psi)}} \\
& =\int_{0}^{x}\left(x-x_{1}\right) d x_{1} \int_{0}^{2 \pi} \frac{d \psi}{2 \pi} \frac{\frac{r_{1} r_{2}}{p_{1} p_{2}} \cos \psi+1}{\sqrt{x_{1}^{2}+R^{2}(\psi)}}
\end{aligned}
$$

Using the expansion eq 27 for this integrand, the $\Psi$ integral vanishes for all terms except $n=0$, so that eq 34 becomes identical with eq 29 , thus proving that the latter corresponds to current sheets.

It is useful to notice the two following partial differential equations satisfied by the components $\omega_{\theta}$ and $\omega_{x}$, which are derivable from the definition 29 together with eq 24 .

$$
\begin{gathered}
\Delta^{2} \omega_{x}=\left(D_{x_{1}}^{2}+D_{r_{1}}+\frac{1}{r_{1}} D_{r_{1}}\right) \omega_{x}=\left(D_{x}^{2}+D_{r_{2}}^{2}+\frac{1}{r_{2}} D_{r_{2}}\right) \omega_{x}=\frac{\phi_{0}\left(k_{0}\right)}{\sqrt{r_{1} r_{2}}} \\
\left(\Delta^{2}-\frac{2}{r_{1}} D_{r_{1}}\right) \omega_{\theta}=\left(D_{x}^{2}+D_{x_{1}}-\frac{1}{r_{1}} D_{r_{1}}\right) \omega_{\theta} \\
=\left(D_{x}^{2}+D_{r_{2}}^{2}-\frac{1}{r_{2}} D_{r_{2}}\right) \omega_{\theta}=\frac{\sqrt{r_{1} r_{2}}}{p_{1} p_{2}} \phi_{1}\left(k_{0}\right)
\end{gathered}
$$

\section{THE PRINCIPAL TERMS $\omega_{\theta}$ AND $\omega_{\theta}^{\prime}$}

The equation 29 gives

where

$$
\omega_{\theta}(x)=x \omega_{\theta}^{\prime}(x)-\frac{\sqrt{r_{1} r_{2}}}{p_{1} p_{2}} \int_{0}^{x} x_{1} \phi_{1}\left(k_{1}\right) d x_{1}
$$

Now, by eq 22

$$
\omega_{\theta}^{\prime}(x)=\frac{\sqrt{r_{1} r_{2}}}{p_{1} p_{2}} \int_{0}^{x} \phi_{1}\left(k_{1}\right) d x_{1}
$$

$$
\frac{1}{k_{1}^{2}}-\frac{1}{k_{0}^{2}}=\frac{x_{1}^{2}}{4 r_{1} r_{2}}
$$

so that

$$
x_{1} d x_{1}=-4 r_{1} r_{2} \frac{d k_{1}}{k_{1}^{3}}
$$


Hence

By eq 18

$$
\frac{-\sqrt{r_{1} r_{2}}}{p_{1} p_{2}} \int_{0}^{x} x_{1} \phi_{1}\left(k_{1}\right) d x_{1}=\frac{-4\left(r_{1} r_{2}\right)^{\frac{3}{2}}}{p_{1} p_{2}} \int_{k}^{k_{0}} \phi_{1}\left(k_{1}\right) \frac{d k_{1}}{k_{1}^{3}} .
$$

Hence

$$
\begin{aligned}
\frac{\phi_{1}\left(k_{1}\right)}{k^{3}}= & =\frac{d}{3} \frac{d}{d k_{1}}\left[\frac{1-k^{2}}{k} \phi_{1}{ }^{\prime}(k)\right]=\frac{1}{6} \frac{d}{d k_{1}}\left\{\phi_{0}\left(k_{1}\right)-\left(\frac{2}{k_{1}^{2}}-1\right) \phi_{1}\left(k_{1}\right)\right\} \\
& =\frac{1}{3 \pi} \frac{d}{d k_{1}}\left[\frac{K\left(k_{1}\right)}{k_{1}}-\left(\frac{2}{k_{1}^{2}}-1\right) \frac{K\left(k_{1}\right)-E\left(k_{1}\right)}{k_{1}}\right]
\end{aligned}
$$

$$
\frac{-\sqrt{r_{1} r_{2}}}{p_{1} p_{2}} \int_{0}^{x} x_{1} \phi_{1}\left(k_{1}\right) d x_{1}=\frac{4\left(r_{1} r_{2}\right)^{\frac{2}{2}}}{3 \pi p_{1} p_{2}}\left[\frac{K}{k}-\left(\frac{2}{k^{2}}-1\right)\left(\frac{K-E}{k}\right)\right]
$$

minus the same function of $k_{0}$, which, being independent of $x$, may be discarded from the $\omega$ function as it always cancels from eq 13 . Hence, placing

$$
\frac{1}{\pi p_{1} p_{2}}=\frac{4 \pi N_{1} N_{2}}{l_{1} l_{2}}
$$

$\omega_{\theta}(x)=x \omega_{\theta}^{\prime}(x)$

$$
+\frac{2 \pi N_{1} N_{2}}{l_{1} l_{2}} \cdot\left(\frac{4 r_{1} r_{2}}{3}\right) \sqrt{x^{2}+\left(r_{1}+r_{2}\right)^{2}}\left[K-\left(\frac{2}{k^{2}}-1\right)(K-E)\right]
$$

To find $\omega_{\theta}^{\prime}(x)$ we use eq 16 .

$$
\begin{aligned}
\omega_{\theta}^{\prime}(x) & =\frac{\sqrt{r_{1} r_{2}}}{\pi p_{1} p_{2}} \int_{0}^{\frac{\pi}{2}} \cos 2 \theta d \theta \int_{0}^{x} \frac{k_{1} d x_{1}}{\sqrt{1-k_{1}^{2} \cos ^{2} \theta}} \\
& =\frac{2 r_{1} r_{2}}{\pi p_{1} p_{2}} \int_{0}^{\frac{\pi}{2}} \cos 2 \theta d \theta \int_{0}^{x} \frac{d x_{1}}{\sqrt{x_{1}^{2}+R^{2}(2 \theta)}} \\
& =\frac{2 r_{1} r_{2}}{\pi p_{1} p_{2}} \int_{0}^{\frac{\pi}{2}} \cos 2 \theta \log \left[\frac{x+\sqrt{x^{2}+R^{2}(2 \theta)}}{R(2 \theta)}\right] d \theta .
\end{aligned}
$$

Integrating this by parts gives

$$
\begin{aligned}
\omega_{\theta}^{\prime}(x) & =\frac{2\left(r_{1} r_{2}\right)^{2} x}{\pi p_{1} p_{2}} \int_{0}^{\frac{\pi}{2}} \frac{\sin ^{2} 2 \theta d \theta}{R^{2}(2 \theta) \sqrt{x^{2}+R^{2}(2 \theta)}} \\
& =\frac{x \sqrt{r_{1} r_{2}}}{4 \pi p_{1} p_{2}} k k_{0}^{2} \int_{0}^{\frac{\pi}{2}} \frac{\sin ^{2} 2 \theta d \theta}{\left(1-k_{0}^{2} \cos ^{2} \theta\right) \sqrt{1-k^{2} \cos ^{2} \theta}} \\
& =\frac{\sqrt{r_{1} r_{2}}}{\pi p_{1} p_{2}} x k \int_{0}^{\frac{\pi}{2}} \frac{\sin ^{2} \theta\left(1-\sin ^{2} \theta\right) d \theta}{\left(\frac{1}{k_{0}^{2}}-\sin ^{2} \theta\right) \sqrt{1-k^{2} \sin ^{2} \theta}}
\end{aligned}
$$


so that

$$
\begin{aligned}
& =\frac{\sqrt{r_{1} r_{2}}}{\pi p_{1} p_{2}} x \int_{0}^{\frac{\pi}{2}} \frac{d \theta}{\sqrt{1-k^{2} \sin ^{2} \theta}}\left\{\frac{1-\left(1-k^{2} \sin ^{2} \theta\right)}{k}\right. \\
& +\frac{k\left(1-k_{0}^{2}\right)}{k_{0}^{2}}\left[1-\frac{1}{1-k_{0}^{2} \sin ^{2} \theta}\right] \text {, }
\end{aligned}
$$

where

$$
\omega_{\theta}^{\prime}(x)=\frac{\left(r_{1}+r_{2}\right) k_{0} k}{2 \pi p_{1} p_{2}} x,\left\{\frac{K-E}{k^{2}}+\frac{k_{0}^{\prime 2}}{k_{0}^{2}}[K-\Pi]\right\}
$$

$$
\begin{aligned}
\Pi & =\int_{0}^{\frac{\pi}{2}} \frac{d \theta}{\left(1-k_{0}^{2} \sin ^{2} \theta\right) \sqrt{1-k^{2} \sin ^{2} \theta}} \\
& =\int_{0}^{K} \frac{d u}{1-k_{0}^{2} \operatorname{sn}^{2}(u, k)}=K+\int_{0}^{K} \frac{k_{0}^{2} \operatorname{sn}^{2} u}{1-k_{0}^{2} \operatorname{sn}^{2} u} d u .
\end{aligned}
$$

The equation may also be put in the form

$$
\omega_{\theta}^{\prime}(x)=\frac{2 \pi N_{1} N_{2}}{l_{1} l_{2}}\left\{x \sqrt{x^{2}+\left(r_{1}+r_{2}\right)^{2}}(K-E)+\frac{x\left(r_{1}-r_{2}\right)^{2}}{\sqrt{x^{2}+\left(r_{1}+r_{2}\right)^{2}}}[K-\Pi]\right\}
$$

For computing, it is sometimes preferable to introduce the Jacobian zeta-function, $\mathrm{Z}$.

$$
K-\Pi=\frac{k_{0}}{k_{0}^{\prime} \sqrt{k_{0}^{2}-k^{2}}}\left[K \mathrm{Z}\left(\theta_{0}, k^{\prime}\right)+\frac{\pi F\left(\theta_{0}, k^{\prime}\right)}{2 K^{\prime}}-\frac{\pi}{2}\right]
$$

where

$$
0<\theta_{0}=\sin ^{-1} \frac{k_{0}^{\prime}}{k^{\prime}}<\frac{\pi}{2}
$$

Hence

$$
\begin{aligned}
\omega_{\theta}^{\prime}(x)=\frac{2 \pi N_{1} N_{2}}{l_{1} l_{2}}\left\{x \sqrt{x^{2}+\left(r_{1}+r_{2}\right)^{2}}(K-E)\right. \\
\left. \pm\left|r_{1}^{2}-r_{2}^{2}\right|\left[K \mathrm{Z}\left(\theta_{0}, k^{\prime}\right)+\frac{\pi F\left(\theta_{0}, k^{\prime}\right)}{2 K^{\prime}}-\frac{\pi}{2}\right]\right\}
\end{aligned}
$$

Where the plus sign belongs with positive $x$ and the minus with negative $x$, the bracket multiplied by \pm vanishing when $x=0$. (Also the absolute value $\left|r_{1}^{2}-r_{2}{ }^{2}\right|$ must be observed.) The zeta-function is expressible in terms of $E\left(\theta, k^{\prime}\right)$ and $F\left(\theta, k^{\prime}\right)$ for

$$
\mathrm{Z}\left(\theta, k^{\prime}\right)=E\left(\theta, k^{\prime}\right)-\frac{E^{\prime}}{K^{\prime}} F\left(\theta, k^{\prime}\right)
$$

Using the AGM method of computation, ${ }^{1}$ and finding $a_{n}, b_{n}$, and $c_{n}$ by the formulas

\footnotetext{
${ }^{1}$ L. V. King, On the Direct Numerical Calculation of Elliptic functions and Integrals, page 8.
} 


$$
\left.\begin{array}{c}
a_{n}=\frac{1}{2}\left(a_{n-1}+b_{n-1}\right), b_{n}=\sqrt{a_{n-1} b_{n-1}}, c_{n}=\frac{1}{2}\left(a_{n-1}-b_{n-1}\right) \\
\tan \left(\theta_{n+1}-\theta_{n}\right)=\frac{b_{n}}{a_{n}} \tan \theta_{n}
\end{array}\right\},
$$

and starting with the initial values

then

$$
a_{0}=1, b_{0}=k, c_{0}=k^{\prime}, \text { and } \theta_{0}=\sin ^{-1} \frac{k_{0}^{\prime}}{k^{\prime}}
$$

$\frac{\pi F\left(\theta_{0}, k^{\prime}\right)}{2 K^{\prime}}=\frac{\theta_{n}}{2^{n}}$ and $\mathrm{Z}\left(\theta_{0}, k^{\prime}\right)=c_{1} \sin \theta_{1}+c_{2} \sin \theta_{2} \ldots c_{n} \sin \theta_{n}$.

Using the initial values $a_{0}=1, b_{0}=k^{\prime}$, and $c_{0}=k$,

gives

$$
K=\frac{\pi}{2 a_{n}} \text { and } K-E=\left(c_{0}^{2}+2 c_{1}^{2}+4 c_{2}^{2} \ldots 2^{n} c_{n}^{2}\right) \frac{K}{2}
$$

The formulas $41^{\prime}$ are the result of successive applications of Landen's transformation which increases the amplitude and decreases the modulus. Hence, they will be most suitable when $k^{\prime}$ is small.

If, however, $k^{\prime}$ is large it is easier to work the transformation in the opposite direction of increasing modulus, in which case the formula is

where

$$
K \mathrm{Z}\left(\theta_{0}, k^{\prime}\right)+\frac{\pi F\left(\theta_{0}, k\right)^{\prime}}{2 K^{\prime}}-\frac{\pi}{2}=-\frac{\pi}{2}\left(1-\sin \psi_{n}\right)+K \sum_{2}\left(\psi_{n}, c_{n}\right),
$$

and

$$
a_{0}=1, b_{0}=k^{\prime}, c_{0}=k
$$

$$
\left.\sin \left(2 \psi_{n+1}-\psi_{n}\right)=\frac{b_{n}}{a_{n}} \sin \psi_{n} \text { (beginning with } \psi_{0}=\theta_{0}\right)
$$

and

$$
\begin{gathered}
\sum_{2}\left(\psi_{n}, c_{n}\right)=2 c_{2} \frac{\tan \left(2 \psi_{2}-\psi_{1}\right)}{\cos \left(2 \psi_{3}-\psi_{2}\right)}+6 c_{3} \frac{\tan \left(2 \psi_{3}-\psi_{2}\right)}{\cos \left(2 \psi_{4}-\psi_{3}\right)}+14 c_{4} \frac{\tan \left(2 \psi_{4}-\psi_{3}\right)}{\cos \left(2 \psi_{5}-\psi_{4}\right)} \\
+\ldots+2\left(2^{1+n}-1\right) c_{n+2} \frac{\tan \left(2 \psi_{n+2}-\psi_{n+1}\right)}{\cos \left(2 \psi_{n+3}-\psi_{n+2}\right)} \ldots .
\end{gathered}
$$

King ${ }^{2}$ also gives an alternative to this with the same $a_{n}, b_{n}$, and $c_{n}$, and the same recurrence formula between $\psi_{n+1}$ and $\psi_{n}$, as above, except that it starts with

$$
\sin ^{2} \psi_{0}=\frac{1}{k^{\prime 2}}\left(1-\frac{k^{2}}{k_{0}^{2}}\right)=\frac{x^{2}}{x^{2}+\left(r_{1}-r\right)^{2}}
$$

${ }^{1}$ There is evidently a misprint in King's formula 7 see eq 75 or 75 defining $\underset{2}{\Sigma}\left(\psi_{n} c_{n}\right)$. The general term should contain the factor $2\left(2^{1+n}-1\right) c_{n+2}$ and not $2\left(2^{n}-1\right) c_{n+2}$. 
in which case

$$
\Pi=\frac{2 K}{k^{\prime 2}} \frac{\cos \left(2 \psi_{1}-\psi_{0}\right)}{\sin 2 \psi_{0}}\left[a_{n} \sin \psi_{n}+\sum_{2}\left(\psi_{n}, c_{n}\right)\right]
$$

To interpret the function $\omega_{\theta}^{\prime}(x)$ in eq 39 it will be found that if the factor $N_{2} / l_{2}=1 / 2 \pi p_{2}$ is omitted and $x$ replaced by $l_{1}$ in eq. 39 the result is the mutual inductance between the helix $h_{1}$ (or its sheet) and a circle of radius $r_{2}$ in its end plane. This is found from eq 13 by letting $l_{2} \rightarrow 0$. The formulas for $\omega_{\theta}(x)$ and $\omega_{\theta}^{\prime}(x)$ when used in eq 13 or $13^{\prime}$ are equivalent to the formulas derived by Jones ${ }^{3}$ for the mutual inductance of two sheets and the force between them. The principal part of the force comes from the $\omega_{\theta}^{\prime}$ function and is, by eq $13^{\prime}$,

$$
f_{\theta}=\left[\omega_{\theta}^{\prime}\left(x_{i_{2}}-x_{i_{1}}\right)-\omega_{\theta}^{\prime}\left(x_{i_{2}}-x_{e_{1}}\right)\right]-\left[\omega_{\theta}^{\prime}\left(x_{e_{2}}-x_{i_{1}}\right)-\omega_{\theta}^{\prime}\left(x_{e_{2}}-x_{e_{1}}\right)\right] .
$$

By the above interpretation of the function $\omega_{\theta}^{\prime}(x)$ it is evident that the first bracket is $N_{2} / l_{2}$ times the mutual inductance between helix $h_{1}$ (or its sheet) and the initial circle of sheet 2 (in the plane $x=x_{i_{2}}$ ). The second bracket is $N_{2} / l_{2}$ times the mutual inductance between $h_{1}$ and the end circle of sheet 2 (in the plane $x=x_{e_{2}}$ ).

\section{EFFECT OF AXIAL CURRENT IN HELICES AND LEAD WIRES}

From eq 29

$$
\begin{aligned}
\omega_{x}(x) & =\frac{x}{\sqrt{r_{1} r_{2}}} \int_{0}^{x} \phi_{0}\left(k_{1}\right) d x_{1}-\frac{1}{\sqrt{r_{1} r_{2}}} \int_{0}^{x} x_{1} \phi_{0}\left(k_{1}\right) d x_{1}=x \omega_{x}^{\prime}(x) \\
& -\frac{1}{\sqrt{r_{1} r_{2}}} \int_{0}^{x} \phi_{0}\left(k_{1}\right) x_{1} d x_{1} \bullet
\end{aligned}
$$

Now, by eq 19 and 22

$$
\begin{aligned}
\frac{1}{\sqrt{r_{1} r_{2}}} \int_{0}^{x} \phi_{0}\left(k_{1}\right) x_{1} d x_{1} & =\frac{1}{\pi \sqrt{r_{1} r_{2}}} \int_{0}^{x} k_{1} K\left(k_{1}\right) x_{1} d x_{1}=\frac{4 \sqrt{r_{1} r_{2}}}{\pi} \int_{k}^{k_{0}} \frac{K\left(k_{1}\right)}{k_{1}{ }^{2}} d k_{1} \\
& =\frac{4 \sqrt{r_{1} r_{2}}}{\pi}\left[\frac{E(k)}{k}-\frac{E\left(k_{0}\right)}{k_{0}}\right] .
\end{aligned}
$$

Hence, we may take

$$
\omega_{x}(x)=x \omega_{x}^{\prime}(x)-\frac{4 \sqrt{ } r_{1} r_{2}}{\pi} \frac{E(k)}{k},
$$

dropping the term in $k_{0}$, which is independent of $x$, and therefore always cancels from eq 13. 
To find $\omega_{x}^{\prime}(x)$ we may use eq 17

$$
\begin{aligned}
\omega_{x}^{\prime}(x) & =\frac{1}{\sqrt{r_{1} r_{2}}} \int_{0}^{x} \phi_{0}\left(k_{1}\right) d x_{1}=\frac{1}{\pi} \sum_{s=0}^{\infty} \frac{\Gamma^{2}\left(s+\frac{1}{2}\right)}{\Gamma^{2}(s+1)}\left(4 r_{1} r_{2}\right)^{s} \int_{0}^{x} \frac{d x_{1}}{\left[x_{1}^{2}+\left(r_{1}+r_{2}\right)^{2}\right]^{s+\frac{1}{2}}} \\
& =\frac{1}{2} \log \frac{1+\sin \gamma}{1-\sin \gamma}+\sin \gamma S\left(\gamma, k_{0}^{2}\right)
\end{aligned}
$$

where

and

$$
\gamma=\tan ^{-1} \frac{x}{r_{1}+r_{2}}
$$

$$
S\left(\gamma, k_{0}\right)=\frac{1}{\pi} \sum_{s=1}^{\infty} \frac{\Gamma^{2}\left(s+\frac{1}{2}\right)}{\Gamma^{2}(s+1)} k_{0}^{2 s} F\left(1-s, \frac{1}{2}, \frac{3}{2}, \sin ^{2} \gamma\right)
$$

Now, $\sin \gamma S$ is, in general, small compared to the logarithm, except when $\gamma=0$, in which case they both vanish; but as $x$ becomes large, $\gamma$ approaches $\pi / 2$, and the logarithm becomes infinite, while the series approaches the finite limit $S\left(\frac{\pi}{2}, k_{0}\right)=\log \frac{2}{1+k_{0}^{\prime}}$, which vanishes if $k_{0}=0$, and has its greatest value, $\log 2=0.69$, when $k_{0}=1$. Also $S$ vanishes when $k_{0}=0$, so that, since the term $\sin \gamma S\left(\gamma_{1} k_{0}\right)$ is a small correction to a second-order term, a good approximation is obtained (sufficient for all cases with which this paper is concerned) by taking

so that

$$
\sin \gamma S\left(\gamma, k_{0}\right)=\sin \gamma \log \frac{2}{1+k_{0}^{\prime}},
$$

$$
\omega_{x}^{\prime}(x)=\log \left[\frac{x+\sqrt{x^{2}+\left(r_{1}+r_{2}\right)^{2}}}{r_{1}+r_{2}}\right]+\frac{x}{\sqrt{x^{2}+\left(r_{1}+r_{2}\right)^{2}}} \log \left(\frac{r_{1}+r_{2}}{r_{m}}\right),
$$

where $r_{m}$ is the greater of $r_{1}$ and $r_{2}\left(\right.$ since $\left.\frac{2}{1+k_{0}^{\prime}}=\frac{r_{1}+r_{2}}{r_{m}}\right)$. It may be noted that the method used to obtain $\int_{0}^{x} \phi_{1}\left(k_{1}\right) d x_{1}$ in the preceding section leads to

$$
\int_{0}^{x} \phi_{0}\left(k_{1}\right) d x_{1}=\frac{1}{\pi} \int_{0}^{\pi} \log \left[\frac{x+\sqrt{x^{2}+R^{2}(\theta)}}{R(\theta)}\right] d \theta .
$$

An approximation similar to this may be obtained from eq 17 in the form

$$
\frac{1}{\sqrt{r_{1} r_{2}}} \int_{0}^{x} \phi_{n}\left(k_{1}\right) d x_{1}=\sin \gamma k_{0}^{2 n} S_{n}\left(\gamma, k_{0}\right)
$$


where

$$
S_{n}\left(\gamma, k_{0}\right)=\frac{1}{\pi} \sum_{s=0}^{\infty} k_{0}^{2 s} \frac{\Gamma^{2}\left(s+n+\frac{1}{2}\right)}{\Gamma(s+1) \Gamma(s+2 n+1)} F\left(1-s-n, \frac{1}{2}, \frac{3}{2}, \sin ^{2} \gamma\right) .
$$

when

$$
\sin ^{2} \gamma=1, F\left(1-s-n, \frac{1}{2}, \frac{3}{2}, 1\right)=\frac{\sqrt{\pi}}{2} \frac{\Gamma(s+n)}{\Gamma\left(s+n+\frac{1}{2}\right)},
$$

so that

$$
\begin{aligned}
& k_{0}^{2 n} S_{n}\left(\frac{\pi}{2}, k_{0}\right)=\frac{1}{2 n}\left(\frac{k_{0}}{2}\right)^{2 n} F\left(n, n+\frac{1}{2}, 2 n+1, k_{0}^{2}\right) \\
& \begin{aligned}
=\frac{1}{2 n}\left(\frac{k_{0}}{1+k_{0}^{\prime}}\right)^{2 n} & =\frac{1}{2 n}\left(\frac{r_{1}}{r_{2}}\right)^{n} \text { if } r_{2}>r_{1} \\
& =\frac{1}{2 n}\left(\frac{r_{2}}{r_{1}}\right)^{n} \text { if } r_{2}<r_{1} .
\end{aligned}
\end{aligned}
$$

Hence an approximation sufficient for use in second order terms is obtained by placing

$$
k_{0}^{2 n} \sin \gamma S_{n}\left(\gamma, k_{0}\right)=\frac{\sin \gamma}{2 n}\left(\frac{k_{0}}{1+k_{0}^{\prime}}\right)^{2 n},
$$

so that the following approximation analagous to eq. 43 is obtained:

$$
\frac{2}{\sqrt{r_{1} r_{2}}} \int_{0}^{x} \phi_{n}\left(k_{1}\right) d x_{1}=\frac{x}{\sqrt{x^{2}+\left(r_{1}+r_{2}\right)^{2}}} \frac{1}{n}\left(\frac{r_{1}}{r_{2}}\right)^{n} \text { if } n>0 \text { and } r_{2}>r_{1} .
$$

When the lead wire of length $l_{1}$ for the return current of $h_{1}$ lies parallel to a generator of the current sheet but at a slightly greater distance from the axis, say $r_{l_{1}}=r_{1}+\Delta r_{1}$, the mutual inductance between this lead wire, $l_{1}$, and the helix, $h_{2}$, is (as far as second-order terms are concerned) equal to that between $l_{1}$ and the current sheet of $h_{2}$, the corresponding $\omega$-function being

$$
-\omega_{x}\left(x, r_{l_{1}}, r_{2}\right)=-\omega_{x}\left(x, r_{1}, r_{2}\right)-\Delta r_{1} D_{r_{1}} \omega_{x}\left(x, r_{1}, r_{2}\right) .
$$

If the lead wire of No. 2 is similarly situated at a distance $r_{l_{2}}=r_{2}+\Delta r_{2}$ its mutual inductance with $h_{1}$ has the $\omega$-function

$$
-\omega_{x}\left(x, r_{1}, r_{l_{2}}\right)=-\omega_{x}\left(x, r_{1}, r_{2}\right)-\Delta r_{2} D_{r_{2}} \omega_{x}\left(x, r_{1} r_{2}\right) .
$$

This arrangement, of course, implies that the number of turns, $N_{1}$ and $N_{2}$, are integers, so that the azimuths of the two lead wires are $\theta_{i_{1}}$ and $\theta_{t_{2}}$. Their mutual inductance with each other is determined by the $\omega$-function, $\omega_{l_{1} l_{2}}$, as in eq 13 , and their force by $\omega_{l_{l} l_{2}}^{\prime}$, as in eq $13^{\prime}$, where

$$
\begin{array}{r}
\omega_{l_{1} l_{2}}\left(x, \alpha, r_{l_{1}}, r_{l_{2}}\right) \\
118273-39-9
\end{array}
$$




$$
\omega_{l_{1} l_{2}}^{\prime}\left(x, \alpha, r_{l_{1}}, r_{l_{2}}\right)=\log \frac{\sqrt{x^{2}+R_{l_{1} l_{2}}^{2}(\alpha)}+x}{R_{l_{1} l_{2}}(\alpha)}=\int_{0}^{x} \frac{d x_{1}}{\sqrt{x_{1}^{2}+R_{l_{1} l_{2}}^{2}(\alpha)}}
$$

where

$$
\begin{aligned}
\alpha & =\theta_{i_{2}}-\theta_{i_{1}} \\
R_{l_{1} l_{2}}^{2}(\alpha) & =r_{l_{1}}^{2}+r_{l_{2}}^{2}-2 r_{l_{1}} r_{l_{2}} \cos \alpha .
\end{aligned}
$$

The additive constant, $R_{l_{1} l_{2}}$, has been omitted from eq 45 , being independent of $x$, so that it cancels from eq 13 . When the radial distances, $\Delta r_{1}$ and $\Delta r_{2}$, of the lead wires from the helices are small, the axial currents in the lead wires and helices almost compensate in their effects upon inductance and force, the residual being a very small term, depending upon the azimuth, $\alpha$, of the coils. If $\bar{\omega}_{x}(x, \alpha)$ represents the total $\omega$-function due to $x$-components of current in lead wires and helices, then

$$
\begin{aligned}
\bar{\omega}_{x}(x, \alpha) & =\omega_{x}\left(x, r_{1}, r_{2}\right)+\omega_{l_{1} l_{2}}\left(x, \alpha, r_{1}+\Delta r_{1}, r_{2}+\Delta r_{2}\right) \\
& -\omega_{x}\left(x, r_{1}+\Delta r_{1}, r_{2}\right)-\omega_{x}\left(x, r_{1}, r_{2}+\Delta r_{2}\right) .
\end{aligned}
$$

When $\Delta r_{1}$ and $\Delta r_{2}$ are small, this becomes $\bar{\omega}_{x}(x, \alpha)=\omega_{l_{1} l_{2}}\left(x, \alpha, r_{l_{1}}, r_{l_{2}}\right)-\omega_{x}\left(x, r_{1}, r_{2}\right)-\left(\Delta r_{1} D_{r_{1}}+\Delta r_{2} D_{r_{2}}\right) \omega_{x}\left(x, r_{1}, r_{2}\right)$. Since, however, these $\omega$-functions are themselves of second order, the terms in $\Delta r$ are smaller than second order and therefore negligible. Hence, for this arrangement of lead wires, the $\omega$-function and its derivative, which takes account of all axial components of current in helices and lead wires, are given by

$$
\bar{\omega}_{x}(x, \alpha)=x \omega_{x}^{\prime}(x, \alpha)+\frac{4 \sqrt{r_{1} r_{2}}}{\pi} \frac{E}{k}-\sqrt{x^{2}+R^{2}(\alpha)}
$$

where

$$
\bar{\omega}_{x}^{\prime}(x, \alpha)=\log \frac{x+\sqrt{x^{2}+R^{2}(\alpha)}}{R(\alpha)}-\omega_{x}^{\prime}(x)=\sum_{n=1}^{\infty} b_{n} \cos n \alpha,
$$

the constant term in this Fourier's cosine series being zero. The coefficients $b_{n}$ for $n>0$ are given by

$$
\begin{aligned}
b_{n} & =\frac{2}{\sqrt{r_{1} r_{2}}} \int_{0}^{x} \phi_{n}\left(k_{1}\right) d x_{1}= \\
& =\frac{x}{\sqrt{x^{2}+\left(r_{1}+r_{2}\right)^{2}} n}\left(\frac{r_{1}}{r_{2}}\right)^{\mathrm{n}}
\end{aligned}
$$

by the approximation in eq. 45 , if $r_{2}>r_{1}$. On summing this 'Fourier's series we find the following finite form as an approximation for eq $46^{\prime}$

$$
\bar{\omega}^{\prime}(x, \alpha)=\frac{x}{\sqrt{x^{2}+\left(r_{1}+r_{2}\right)^{2}}} \log \frac{r_{2}}{R(\alpha)}
$$

when $r_{2}>r_{1}$. 


\section{THE AZIMUTHAL TERMS}

To integrate eq 30 , we may place (by reference to eq $10^{\prime}$ )

$$
\cos n \psi_{x}=e^{i n \psi_{x}}=e^{i n\left(\theta_{x}-\frac{x}{p_{1}}\right)+\frac{i n x_{1}}{p_{1}}+\left(\frac{1}{p_{1}}-\frac{1}{p_{2}}\right) i n x_{2},}
$$

with the understanding that the real part of the result is to be taken. Using the abbreviation

$$
J(n, s)=J_{n-1}\left(r_{1} s\right) J_{n-1}\left(r_{2} s\right)+J_{n+1}\left(r_{1} s\right) J_{n+1}\left(r_{2} s\right)+\frac{2 p_{1} p_{2}}{r_{1} r_{2}} J_{n}\left(r_{1} s\right) J_{n}\left(r_{2} s\right) .
$$

eq 30 becomes, by use of eq 25 ,

$$
\omega_{n}\left(x, \theta_{x}\right) \equiv \frac{r_{1} r_{2}}{p_{1} p_{2}} e^{i n\left(\theta_{x}-\frac{x}{p_{1}}\right)} \int_{0}^{\infty} d s J(n, s) \int_{0}^{x} e^{i n\left(\frac{1}{p_{1}}-\frac{1}{p_{2}}\right) x_{2}} d x_{2} \int_{0}^{x_{1}} e^{\frac{i n x_{1}}{p_{1}}-s\left|x_{1}\right|} d x_{1} .
$$

If $x>0$, then $x_{1}$ and $x_{2}$ are both positive in the range of integration of the $x_{1} x_{2}$ double integral, so that $e^{-s\left|x_{1}\right|}=e^{-s x_{1}}$. When $x<0$, then $x_{1}$, and $x_{2}$ are both negative in the range of the integral, so that $e^{-s \mid x_{1} l}=$ $e^{+s x_{1}}$, and the result of the integration in the latter case is found by changing the sign of $s$ in the result of the integration for the first case.

It is thus found that both results are included in the following:

$$
\begin{aligned}
\omega_{n}\left(x, \theta_{x}\right) & =\frac{r_{1} r_{2} e^{i n \theta x}}{n^{2}\left(p_{2}-p_{1}\right)} \int_{0}^{\infty} d s J(n, s)\left\{\frac{p_{2}\left(e^{-\frac{i n x}{p_{2}}}-e^{-s|x|}\right)}{1 \pm \frac{i p_{2} s}{n}}-\frac{p_{1}\left(e^{-\frac{i n x}{p_{1}}}-e^{-s|x|}\right)}{1 \pm \frac{i p_{1} s}{n}}\right\} \\
& =\frac{r_{1} r_{2} e^{i n \theta x}}{n^{2}} \int_{0}^{\infty} d s J(n, s) \\
& \left\{\frac{p_{2} e^{-\frac{i n x}{p_{2}}}}{\left(p_{2}-p_{1}\right)\left(1 \pm \frac{i p_{2} s}{n}\right)}-\frac{p_{1} e^{-\frac{i n x}{p_{1}}}}{\left(p_{2}-p_{1}\right)\left(1 \pm \frac{i p_{1} s}{n}\right)}-\frac{e^{-s|x|}}{\left(1 \pm \frac{i p_{1} s}{n}\right)\left(1+\frac{i p_{2} s}{n}\right)}\right\},
\end{aligned}
$$

where the upper sign belongs with positive $x$ and the lower with negative $x$. 
The real part of this is

$$
\begin{aligned}
& \omega_{n}\left(x, \theta_{x}\right)=\frac{r_{1} r_{2}}{n^{2}} \int_{0}^{\infty} d s J(n, s)\left\{\frac{1}{p_{2}-p_{1}}\left[\frac{p_{2} \cos n\left(\theta_{x}-\frac{x}{p_{2}}\right)}{1+\frac{p_{1}^{2} s^{2}}{n^{2}}}-\frac{p_{1} \cos n\left(\theta_{x}-\frac{x}{p_{1}}\right)}{1+\frac{p_{1}^{2} s^{2}}{n^{2}}}\right]-\frac{\left(1-\frac{p_{1} p_{2} s^{2}}{n^{2}}\right) e^{-s|x|} \cos n \theta_{x}}{\left(1+\frac{p_{1}^{2} s^{2}}{n^{2}}\right)\left(1+\frac{p_{1}^{2} s^{2}}{n^{2}}\right)}\right\} \\
& \pm \frac{r_{1} r_{2}}{n^{3}} \int_{0}^{\infty} \operatorname{sdsJ}(n, s)\left\{\frac{1}{p_{2}-p_{1}}\left[\frac{p_{2}^{2} \sin n\left(\theta_{x}-\frac{x}{p_{2}}\right)}{1+\frac{p_{2}^{2} s^{2}}{n^{2}}}-\frac{p_{1}^{2} \sin n\left(\theta_{x}-\frac{x}{p_{1}}\right)}{1+\frac{p_{1}^{p_{2}} s^{2}}{n^{2}}}\right]-\frac{\left(p_{1}+p_{2}\right) e^{-s|x|} \sin n \theta_{x}}{\left(1+\frac{p_{1}^{2} s^{2}}{n^{2}}\right)\left(1+\frac{p_{s}^{2} s^{2}}{n^{2}}\right)}\right\}
\end{aligned}
$$


The terms in the first integral which do not contain the exponential factor $e^{-s|x|}$ may be discarded, as they will all cancel in eq 13 because, as shown by reference to eq 2 and 11 , the values of $\theta_{x}-x / p_{1}$ will be the same for the first and second $x$-differences, $\left(x e_{2}-x i_{1}\right)$ and $\left(x e_{2}-x e_{1}\right)$. These first and second $x$-values enter eq 13 with opposite signs. Similarly, the values of $\theta_{x}-x / p_{1}$ are the same for the third and fourth $x$-values, $x_{i_{2}}-x_{e_{1}}$ and $x_{i_{2}}-x_{i_{1}}$, which enter eq 13 with opposite signs. Hence the term in eq $47, \cos n\left(\theta_{x}-x / p_{1}\right)$, disappears by the second $x$-term canceling the first and the fourth canceling the third. It is also found that $\cos n\left(\theta_{x}-x / p_{2}\right)$ disappears by the fourth canceling the first and the third canceling the second. The corresponding terms in the second integral, $\sin n\left(\theta_{x}-x / p_{1}\right)$ and $\sin n\left(\theta_{x}-x / p_{1}\right)$, do not always cancel because of the \pm factor which has the sign of $x$. If the associated current sheets are wholly external to each other the cancelation is complete, but if one lies wholly or partly within the other the terms $\sin n\left(\theta_{x}-x / p_{1}\right)$ and $\sin n\left(\theta_{x}-x / p_{2}\right)$ of eq 47 do not disappear from eq 13.

Hence eq 47 may be written

$$
\begin{gathered}
\omega_{n}\left(x, \theta_{x}\right)=-\frac{r_{1} r_{2}}{n_{2}} \cos n \theta_{x} \int_{0}^{\infty} \frac{e^{-s|x|} J(n, s)\left(1-\frac{p_{1} p_{2}}{n_{2}} s^{2}\right)}{\left(1+\frac{p_{1}^{2} s^{2}}{n^{2}}\right)\left(1+\frac{p_{2}^{2} s^{2}}{n^{2}}\right)} d s \\
\mp \frac{r_{1} r_{2}\left(p_{1}+p_{2}\right)}{n^{3}} \sin n \theta_{x} \int_{0}^{\infty} \frac{s e^{-s|x|} J\left(n_{1} s\right)}{\left(1+\frac{p_{1}^{2} s^{2}}{n^{2}}\right)\left(1+\frac{p_{s}^{2} s^{2}}{n^{2}}\right)} d s \pm \omega_{n}^{*}\left(x, \theta_{x}\right),
\end{gathered}
$$

plus terms which cancel in eq 13 , where

$$
\begin{gathered}
\omega_{n}^{*}\left(x, \theta_{x}\right)=\frac{r_{1} r_{2}}{n\left(p_{2}-p_{1}\right)} \\
\left\{\sin n\left(\theta_{x}-\frac{x}{p_{2}}\right) \int_{0}^{\infty} \frac{s J(n, s) d s}{s^{2}+\frac{n^{2}}{p_{2}}}-\sin n\left(\theta_{x}-\frac{x}{p_{1}}\right) \int_{0}^{\infty} \frac{s J(n, s) d s}{s^{2}+\frac{n^{2}}{p_{1}^{2}}}\right\}
\end{gathered}
$$

We may now show that by limiting the application of the results of this section to those cases where the $\omega_{n}^{*}(x)$ are all negligible, we do not impose a serious restriction as far as practical mutual inductances are concerned, with the exception of a bifilar-wound coil in which one of the windings is used as primary, the other as secondary. Such a coil has never been used for absolute measurements, but we see no reason why it should not be used in the future. It would possess certain advantages from the point of view of accurate construction and measurement and would give the greatest possible mutual inductance. The formula for its mutual inductance has never been evaluated with precision as it has for a self inductance, but the problem would present no greater difficulty.

It is easy to foretell that this limitation is that the helices, $h_{1}$ and $h_{2}$ shall not through a finite part of their lengths be separated from each other by a distance which is small (of the order of $p / r$ ). 
If $H_{n}$ denotes the first Hankel's function then

$$
\int_{0}^{\infty} \frac{s J_{m}\left(r_{1} s\right) J_{m}\left(r_{2} s\right) d s}{s^{2}+\frac{n^{2}}{p^{2}}}=\frac{\pi}{2} J_{m}\left(\frac{i n r_{1}}{p}\right) i H_{m}\left(\frac{i n r_{2}}{p}\right) .
$$

when $r_{2}>r_{1}$. In case $r_{2}<r_{1}$, the two are interchanged in this equation. Since $r_{1} / p$ and $r_{2} / p$ are large, the asymptotic expansions of $H_{m}$ and $J_{m}$ show that this expression is very close to

$$
\frac{p}{2 n \sqrt{r_{1} r_{2}}} e^{\frac{-n\left|r_{2}-r_{1}\right|}{p}} \text { in cases } r_{2}>r_{1} \text { or } r_{2}<r_{1} \text {. }
$$

Hence, approximately,

$$
\omega_{n}^{*}\left(x, \theta_{x}\right)=\frac{\sqrt{r_{1} r_{2}}}{n^{2}\left(p_{1}-p_{2}\right) i}\left\{p_{2} e^{-\frac{n}{p_{2}}\left[\left|r_{2}-r_{1}\right|+i\left(x-p_{2} \theta x\right)\right]}-p_{1} e^{-\frac{n}{p_{1}}\left[\left|r_{2}-r_{1}\right|+i\left(x-p_{1} \theta_{x}\right)\right]}\right\},
$$

where the real part is to be taken.

When $\mathrm{p}_{2}=p_{1}=p$, this becomes

$$
\omega_{n}^{*}\left(x, \theta_{x}\right)=\frac{\sqrt{r_{1} r_{2}}}{i}\left\{\frac{1}{n^{2}}+\frac{\left|r_{2}-r_{1}\right|+i x}{n p}\right\} e^{-\frac{n}{p}\left[\left|r_{2}-r_{1}\right|+i\left(x-p \theta_{x}\right)\right]}
$$

Since the entire $\omega_{a}$ function with the factor $r_{1} r_{2}$ is of second order in general, compared with the principal term, $\omega_{\theta}$, which has the large factor $\frac{r_{1} r_{2}}{p_{1} p_{2}}$, it is evident that $\sum_{1}^{\infty} \frac{e}{n}-\left.\frac{n}{p}\right|^{\left|r_{2}-r_{1}\right|}$ will be utterly negligible when $r_{2}-r_{1}$ is finite (not a small quantity of the order of $p$ ). Since $\omega_{n}^{*}$ is only different from zero when one coil lies wholly or partly within the other, this means that the two must not approach very close to each other if $\omega_{n}^{*}$ is to be neglected.

The remaining integrals in eq 48 cannot be similarly integrated in finite terms, but it is evident that when $|x|$ is finite (not small of the order $p / r$ ), we may (on account of the convergence factor $e^{-s|x|}$ of the integrand) obtain the principal part of $\omega_{n}$ by placing $p_{1}=p_{2}=0$ in the integrand, as the resulting integrals still converge, even if $r_{1}=r_{2}$. We cannot be content, however, with this restriction on $x$, as we must evaluate $\omega_{n}$ for cases where $x=0$. In this case, if we place $p_{1}=p_{2}=0$, the integrals in eq 48 then converge only if $r_{1} \neq r_{2}$, but if $r_{1}-r_{2}$ is a finite quantity, the principal part of $\omega_{n}$ is thus obtained. The limitation is therefore evident- the helices must be everywhere separated from each other by finite distances. Assuming this to be the case, and noting that the last term in the definition of $J(n, s)$ is negligible, we obtain the result

$$
\omega_{n}\left(x, \theta_{x}\right)=-\frac{\sqrt{r_{1} r_{2}}}{n^{2}}\left[\phi_{n-1}(k)+\phi_{n+1}(k)\right] \cos n \theta_{x}
$$

so that

$$
\omega_{a}(x, \theta x)=-\sqrt{r_{1} r_{2}} \sum_{n=1}^{\infty}\left[\phi_{n-1}+\phi_{n+1}\right] \frac{\cos n \theta_{x}}{n^{2}},
$$


and

$$
\omega_{u}^{\prime}\left(x, \theta_{x}\right)=\frac{x k^{3}}{4 \sqrt{r_{1} r_{2}}} \sum_{n=1}^{\infty}\left[\phi_{n-1}^{\prime}+\phi_{n+1}^{\prime}\right] \frac{\cos n \theta_{x}}{n^{2}}
$$

(In finding the self inductance of a helix, the $\omega_{a}$ rises in rank from a second order to a first order quantity, from which originates the most important correction term-the evaluation of which was the only difficult part of that problem. The same remark would apply to a bifilar mutual inductance). Eq 49 holds of course when $r_{1}=r_{2}$, provided the end planes of the two coils are not close together and the coils wholly external to each other (so that $k$ is not close to unity).

The series 49 and $49^{\prime}$ may be evaluated by finding $\phi_{0}$ and $\phi_{1}$ in terms of elliptic integrals by eq 19, and then finding all other $\phi_{n}$ or $\phi_{n}^{\prime}$ by the recurrence relation 20 or 21 .

Since the series represent second-order terms, it is evident that some simpler approximation will be sufficient. To obtain such, consider the even, continuous function of $\theta, f(\theta)$, which is never negative and is a periodic function of $\theta$, with period $2 \pi$, defined by the series

$$
f(\theta) \equiv \frac{2}{3}-\frac{4}{\pi^{2}} \sum_{n=1}^{\infty} \frac{\cos n \theta}{n^{2}} \text { for all real values of } \theta .
$$

$f(\theta)$ vanishes when $\theta=2 n \pi$, where $n$ is any integer. It has the maximum value unity when $\theta=(2 n+1) \pi$. It consists of a succession of parabolic ares for

$$
f(\theta)=\left(2-\frac{\theta}{\pi}\right)_{\pi}^{\theta} \ldots \ldots . . . . \text { when } 0 \leqq \theta \leqq 2 \pi,
$$

and

$$
f(\theta)=\left(4-\frac{\theta}{\pi}\right)\left(\frac{\theta}{\pi}-2\right) \ldots . . . \text { when } 2 \pi \leqq \theta \leqq 4 \pi .
$$

Now,

$$
f\left(\theta+\theta_{x}\right)=\frac{2}{3}-\frac{4}{\pi^{2}} \sum_{n=1}^{\infty} \frac{\cos n\left(\theta+\theta_{x}\right) .}{n^{2}} .
$$

Also from eq 15 one finds

$$
\frac{k}{2} \frac{\cos \theta}{\sqrt{1-k^{2} \cos ^{2} \frac{\theta}{2}}}=\phi_{1}(k)+\sum_{n=1}^{\infty}\left[\phi_{n-1}(k)+\phi_{n+1}(k)\right] \cos n \theta .
$$

Multiplying eq 52 by eq 53 , and integrating the product from $\theta=0$ to $\theta=2 \pi$, gives

$$
\frac{k}{2} \int_{0}^{2 \pi f\left(\theta+\theta_{x}\right) \cos \theta d \theta} \frac{4 \pi}{\sqrt{1-k^{2} \cos ^{2} \frac{\theta}{2}}}=\frac{4 \pi}{3} \phi_{1}(k)-\frac{4}{\pi} \sum_{n=1}^{\infty}\left(\phi_{n-1}+\phi_{n+1}\right) \frac{\cos n \theta_{x}}{n^{2}},
$$


so that eq 49 becomes

$$
\begin{aligned}
\omega_{a}\left(x, \theta_{x}\right) & =-\sqrt{r_{1} r_{2}}\left\{\frac{\pi^{2}}{3} \phi_{1}(k)-\frac{\pi k}{8} \int_{0}^{2 \pi} \frac{f\left(\theta+\theta_{x}\right) \cos \theta d \theta}{\sqrt{1-k^{2} \cos ^{2} \frac{\theta}{2}}}\right\} \\
& =-\sqrt{r_{1} r_{2}}\left\{\frac{\pi^{2}}{3} \phi_{1}-\frac{\pi k}{8} \int_{0}^{2 \pi-\theta_{x}} \frac{f\left(\theta+\theta_{x}\right) \cos \theta d \theta}{\sqrt{1-k^{2} \cos ^{2} \frac{\theta}{2}}}-\frac{\pi k}{8} \int_{2 \pi-\theta_{x}}^{2 \pi} \frac{f\left(\theta+\theta_{x}\right) \cos \theta}{\sqrt{1-k^{2} \cos ^{2} \frac{\theta}{2}}} d \theta\right\}
\end{aligned}
$$

It is sufficient to evaluate this for the range $0 \bar{\gtrless} \theta_{x} \leqq \pi$ since its value for any other range of $\theta_{x}$ is obtainable from this by inspection, remembering that $\omega_{a}$ is an even function of $\theta_{x}$ with period $2 \pi$. (This restriction disappears from the end result.) With this restriction the argument of $f$ in the integral from 0 to $2 \pi-\theta_{x}$ lies in the range $0 \leqq \theta+\theta_{x} \leqq 2 \pi$, so that by eq $51 \mathrm{a}$

$$
f\left(\theta+\theta_{x}\right)=\left(2-\frac{\theta+\theta_{x}}{\pi}\right)\left(\frac{\theta+\theta_{x}}{\pi}\right) .
$$

In the integral last written, as eq $51 \mathrm{~b}$ shows,

$$
f\left(\theta+\theta_{x}\right)=\left(4-\frac{\theta+\theta_{x}}{\pi}\right)\left(\frac{\theta+\theta_{x}}{\pi}-2\right) .
$$

Making these substitutions, and then changing the variable of integration in the last integral to $\theta^{\prime}$, where $\theta=2 \pi-\theta^{\prime}$, gives

$$
\begin{aligned}
\omega_{u}\left(x, \theta_{x}\right)=-\sqrt{r_{1} r_{2}}\left\{\frac{\pi^{2}}{3} \phi_{1}(k)-\frac{\pi k}{8} \int_{0}^{2 \pi-\theta_{x}}\left[2-\frac{\theta_{x}+\theta}{\pi}\right] \frac{\theta_{x}+\theta}{\pi} \frac{\cos \theta d \theta}{\sqrt{1-k^{2} \cos ^{2} \frac{\theta}{2}}}\right. \\
\left.-\frac{\pi k}{8} \int_{0}^{\theta_{x}}\left[2-\frac{\theta_{x}-\theta}{\pi}\right] \frac{\theta_{x}-\theta}{\pi} \sqrt{\sqrt{1-k^{2} \cos ^{2} \frac{\theta}{2}}}\right\}
\end{aligned}
$$

or

$\omega_{a}\left(x, \theta_{x}\right)=-\sqrt{r_{1} r_{2}}\left\{\frac{\pi^{2}}{3} \phi_{1}-\frac{\pi k}{8} \int_{0}^{2 \pi-\theta_{x}} \frac{f\left(\theta_{x}+\theta\right) \cos \theta d \theta}{\sqrt{1-k^{2} \cos ^{2} \frac{\theta}{2}}}-\frac{\pi k}{8} \int_{0}^{\theta_{x}} \frac{f\left(\theta_{x}-\theta\right) \cos \theta d \theta}{\sqrt{1-k^{2} \cos ^{2} \frac{\theta}{2}}}\right\}$,

where both of the arguments $\theta_{x}+\theta$ and $\theta_{x}-\theta$ lie in the positive range of less than $2 \pi$. For this range, instead of the cosine series 50 , we find a sine series for $f(\theta)$

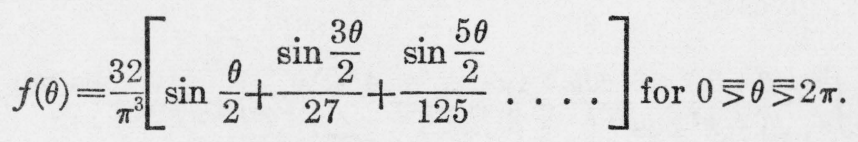

Since $32 / \pi^{3}=1.032$, it is evident that a good approximation for the integrals in eq 54 is given by placing $f(\theta)=\sin \frac{\theta}{2}$ for $0 \leqq \theta \leqq 2 \pi$, that is, 


$$
f\left(\theta_{x}+\theta\right)=\sin \left(\frac{\theta_{x}+\theta}{2}\right) \text { and } f\left(\theta_{x}-\theta\right)=\sin \left(\frac{\theta_{x}-\theta}{2}\right) .
$$

This gives after some simple transformations

$$
\begin{aligned}
\omega_{a}\left(x, \theta_{x}\right)=-\sqrt{r_{1} r_{2}}\left\{\frac{\pi^{2}}{3} \phi_{1}(k)+\right. & \frac{\pi k}{2} \sin \frac{\theta_{x}}{2} \int_{\left(\frac{\pi-\theta_{x}}{2}\right)}^{\frac{\pi}{2}} \frac{\sin \theta \cos 2 \theta}{\sqrt{1-k^{2} \sin ^{2} \theta}} d \theta \\
& \left.+\frac{\pi k}{2} \cos \frac{\theta_{x}}{2} \int_{0}^{\left(\frac{\pi-\theta_{x}}{2}\right)} \frac{\cos \theta \cos 2 \theta}{\sqrt{1-k^{2} \sin ^{2} \theta}} d \theta\right\} .
\end{aligned}
$$

Now,

$$
\begin{aligned}
& k \int \frac{\sin \theta \cos 2 \theta}{\sqrt{1-k^{2} \sin ^{2} \theta}} d \theta=\frac{1}{k^{2}}\left[\log \left(k \cos \theta+\sqrt{1-k^{2} \sin ^{2} \theta}\right)\right. \\
&\left.-k \cos \theta \sqrt{1-k^{2} \sin ^{2} \theta}\right]
\end{aligned}
$$

and

$$
k \int \frac{\cos \theta \cos 2 \theta}{\sqrt{1-k^{2} \sin ^{2} \theta}} d \theta=\frac{1}{k^{2}}\left[-k^{2} \sin ^{-1}(k \sin \theta)+k \sin \theta \sqrt{1-k^{2} \sin ^{2} \theta}\right],
$$

so that

$$
\omega_{a}\left(x, \theta_{x}\right)=-\frac{\sqrt{r_{1} r_{2}} \pi^{2} \phi_{1}(k)}{3}-\frac{\pi \sqrt{r_{1} r_{2}}}{2}\left[\frac{1}{k} \sqrt{1-k^{2} \cos ^{2} \frac{\theta_{x}}{2}}\right.
$$

$\left.-\frac{k^{\prime 2}}{k^{2}} \cos \frac{\theta_{x}}{2} \sin ^{-1}\left(k \cos \frac{\theta_{x}}{2}\right)-\frac{1}{k} \sin \frac{\theta_{x}}{2} \log \frac{k \sin \frac{\theta_{x}}{2}+\sqrt{1-k^{2} \cos ^{2} \frac{\theta_{x}}{2}}}{k^{\prime}}\right]$.

Differentiating this with respect to $x$ gives

$$
\begin{aligned}
& \omega_{a}^{\prime}\left(x, \theta_{x}\right)=\frac{x}{12 \sqrt{r_{1} r_{2}}} \cdot \pi^{2} k^{3} \phi_{1}^{\prime}(k) \\
& +\frac{\pi x}{4 \sqrt{r_{1} r_{2}}}\left\{\cos \frac{\theta_{x}}{2} \sin ^{-1}\left(k \cos \frac{\theta_{x}}{2}\right)-\frac{k\left(1-\frac{k^{2}}{2}\right)}{k^{\prime 2}} \sqrt{1-k^{2} \cos \frac{\theta_{x}}{2}}\right. \\
& \left.+k \sin \frac{\theta_{x}}{2} \log \left[\frac{k \sin \frac{\theta_{x}}{2}+\sqrt{1-k^{2} \cos ^{2} \frac{\theta_{x}}{2}}}{k^{\prime}}\right]\right\}
\end{aligned}
$$

These equations hold for any value of $\theta_{x}$ since they are even periodic functions of $\theta_{x}$, with period $2 \pi$. 
The four azimuthal differences in eq 11 may be written.

$$
\begin{aligned}
& \theta_{c_{2} i_{1}}=\alpha+2 \pi N_{2}, \theta_{e_{2} e_{1}}=\alpha+2 \pi\left(N_{2}-N_{1}\right) \\
& \theta_{i_{2} e_{1}}=\alpha-2 \pi N_{1}, \theta_{i_{2} i_{1}} \equiv \alpha \equiv \theta_{i_{2}}-\theta_{i_{1}}
\end{aligned}
$$

so that when the number of turns, $N_{1}$ and $N_{2}$, are integers, every $\theta_{x}$ may be taken equal to $\alpha$. It is evident that no essential simplification would have resulted in the formulas eq 57 and 58 had we restricted $N_{1}$ and $N_{2}$ to integral values at the beginning.

To obtain an interpretation of the term $\omega_{a}\left(x, \theta_{x}\right)$, let $M\left(x, \theta_{x}, r_{1}, r_{2}\right)$, or, more briefly, $M\left(\theta_{x}\right)$, denote the mutual inductance between the two incomplete circular arcs which are the parts of the two coaxial circles of radii $r_{1} r_{2}$ (the distance between their planes being $x$ ) included between the azimuth planes $\theta=0$ and $\theta=\theta_{x}$.

When the circles are complete we have by eq 23

$$
M(2 \pi)=4 \pi^{2} \sqrt{r_{1} r_{2}} \phi_{1}(k)
$$

In general, if $0<\theta_{x}<2 \pi$

$$
M\left(\theta_{x}\right)=r_{1} r_{2} \int_{0}^{\theta_{x}} d \theta_{1} \int_{0}^{\theta_{x}} d \theta_{2} \frac{\cos \left(\theta_{2}-\theta_{1}\right)}{\sqrt{x^{2}+R^{2}\left(\theta_{2}-\theta_{1}\right)}}
$$

which may be transformed into

$$
\frac{M\left(\theta_{x}\right)}{2 \sqrt{r_{1} r_{2}}}=\int_{0}^{\theta_{x}} d \theta_{2} \int_{0}^{\theta_{2}} \frac{k \cos \theta_{1} d \theta_{1}}{2 \sqrt{1-k^{2} \cos ^{2} \frac{\theta_{1}}{2}}} .
$$

Using the expansion in eq 53 for this integrand gives

$$
\frac{M\left(\theta_{x}\right)}{2 \sqrt{r_{1} r_{2}}}=\frac{\theta_{z}^{2}}{2} \phi_{1}(k)+\sum_{n=1}^{\infty} \frac{\phi_{n-1}(k)+\phi_{n+1}(k)}{n^{2}}\left(1-\cos n \theta_{x}\right) .
$$

Equations 49 and 59 are equivalent to

$$
\omega_{a}\left(x, \theta_{x}\right)=\omega_{a}(x, 0)+\frac{1}{2}\left[M\left(\theta_{x}\right)-\left(\frac{\theta_{x}}{2 \pi}\right)^{2} M(2 \pi)\right] \text { when } 0 \leqq \theta_{x} \leqq 2 \pi .
$$

Since, by eq 57 ,

$$
\omega_{a}(x, 0)=-\frac{M(2 \pi)}{12}-\frac{\pi \sqrt{r_{1} r_{2}} k^{\prime}}{2 k}\left(1-\frac{k^{\prime}}{k} \sin ^{-1} k\right),
$$

this may be written

$$
\begin{aligned}
\omega_{a}\left(x, \theta_{x}\right)=-\frac{M(2 \pi)}{12}+\frac{1}{2}\left[M\left(\theta_{x}\right)-\left(\frac{\theta_{x}}{2 \pi}\right)^{2} M(2 \pi)\right] & \\
& -\frac{\pi \sqrt{r_{1} r_{2}} k^{\prime}}{2 k}\left[1-\frac{k^{\prime}}{k} \sin ^{-1} k\right],
\end{aligned}
$$

which furnishes an interpretation for part of the term $\omega_{a}\left(x, \theta_{x}\right)$, although this form has no advantage in computation since the mutual induc- 
tance $M\left(\theta_{x}\right)$ of the two incomplete circular arcs is not a well-known or tabulated function as in the case $M(2 \pi)$ when the arcs are complete circles. In fact, the foregoing formulas give for it

$$
\begin{aligned}
& M(\theta)=\left(\frac{\theta}{2 \pi}\right)^{2} M(2 \pi)--\pi \sqrt{r_{1} r_{2}} \mid \frac{\sqrt{1-k^{2} \cos ^{2} \frac{\theta}{2}}-k^{\prime}}{k} \\
&-\frac{k^{\prime 2}}{k^{2}}\left[\cos \frac{\theta}{2} \sin ^{-1}\left(k \cos \frac{\theta}{2}\right)-\sin ^{-1} k\right] \\
&-\frac{1}{k^{2}} \sin \frac{\theta}{2} \log \left[\frac{k \sin \frac{\theta}{2}+\sqrt{1-k^{2} \cos ^{2} \frac{\theta}{2}}}{k^{\prime}}\right] .
\end{aligned}
$$

When the series 49 and $49^{\prime}$ are known to converge so rapidly that only the first two terms need be retained, there is no need to use eq 57 and $57^{\prime}$.

\section{THE HELIX EQUIVALENT TO A HELICAL WIRE}

Consider the unit current in wire $w_{1}$ to have the vector volume density whose magnitude is a function of the distance $r_{1}^{\prime}$ from the $x$ axis, say $u_{1}\left(r_{1}^{\prime}\right)$. Its direction is that of the generating helical filament.

An axial plane cuts the wire in a circular section of radius $\rho_{1}$, as specified in eq 5. If $d S_{1}$ is an element of area of this circular section, a helical tube whose (oblique) section is $d S_{1}$ carries the current,

since

$$
d I_{1}=\frac{u_{1}\left(r_{1}^{\prime}\right) d S_{1}}{\sqrt{1+\left(\frac{p_{1}}{r_{1}^{\prime}}\right)^{2}}}
$$

$$
\frac{1}{\sqrt{1+\left(\frac{p_{1}}{r_{1}^{\prime}}\right)^{2}}}
$$

is the cosine of the angle between the normal to $d S_{1}$ and the direction of the current-density vector or tube. The total current carried by the wire is unity, so that

$$
\int \frac{u_{1}\left(r_{1}^{\prime}\right) d S_{1}}{\sqrt{1+\left(\frac{p_{1}}{r_{1}^{\prime}}\right)^{2}}}=1
$$

integrated over the circular section of the wire.

In place of the rectangular coordinates $x_{i_{1}}^{\prime}-x_{i}$ and $r_{1}^{\prime}-r_{1}$ of a point of this section referred to the center of the circle as origin (as used in eq 5), we may use the polar coordinates $\rho_{1}^{\prime}$ and $\phi_{1}$, where

$$
x_{i_{1}}^{\prime}-x_{i_{1}}=\rho_{1}^{\prime} \cos \phi_{1} \text { and } r_{1}^{\prime}-r_{1}=\rho_{1}^{\prime} \sin \phi_{1} \text {, }
$$


in terms of which

$$
d S_{1}=\rho_{1}^{\prime} d \rho_{1}^{\prime} d \phi_{1} .
$$

Expanding the current density, $u_{1}\left(r_{1}^{\prime}\right)=u_{1}\left(r_{1}+\rho_{1}^{\prime} \sin \phi_{1}\right)$, about the value $r_{1}$ (corresponding to the central filament of the wire) by Taylor's theorem and applying the condition 62, we obtain (to the second order, inclusive, in $\rho_{1}$ )

where

$$
d I_{1}=\frac{\rho_{1}^{\prime} d \rho_{1}^{\prime} d \phi_{1}}{\pi \rho_{1}^{2}}\left[1+A_{1} \rho_{1}^{\prime} \sin \phi_{1}+B_{1}\left(\rho_{1}^{\prime 2} \sin ^{2} \phi_{1}-\frac{\rho_{1}^{2}}{4}\right)\right]
$$

$$
A_{1}=\frac{u_{1}^{\prime}\left(r_{1}\right)}{u_{1}\left(r_{1}\right)} \text { and } B_{1}=\frac{u_{1}^{\prime \prime}\left(r_{1}\right)}{2 u_{1}\left(r_{1}\right)} \text {. }
$$

With similar definitions of $A_{2}$ and $B_{2}$, etc., the current, $d I_{2}$, in a helical current tube of the second wire is

so that

$$
d I_{2}=\frac{\rho_{2}^{\prime} d \rho_{2}^{\prime} d \phi_{1}}{\pi \rho_{2}^{2}}\left[1+A_{2} \rho_{2}^{\prime} \sin \phi_{2}+B_{2}\left(\rho_{2}^{\prime \prime} \sin ^{2} \phi_{2}-\frac{\rho_{2}^{2}}{4}\right)\right]
$$

$$
\begin{aligned}
& d I_{1} d I_{2}=\frac{\rho_{1}^{\prime} d \rho_{1}^{\prime} d \phi_{1} \rho_{2}^{\prime} d \rho_{2}^{\prime} d \phi_{2}}{\pi^{2} \rho_{1}^{2} \rho_{2}^{2}}\left\{1+\left(A_{1} \rho_{1}^{\prime} \sin \phi_{1}+A_{2} \rho_{2}^{\prime} \sin \phi_{2}\right)\right. \\
& \left.\quad+B_{1}\left(\rho_{1}^{\prime 2} \sin ^{2} \phi_{1}-\frac{\rho_{1}^{2}}{4}\right)+B_{2}\left(\rho_{2}^{\prime 2} \sin ^{2} \phi_{2}-\frac{\rho_{2}^{2}}{4}\right)+A_{1} A_{2} \rho_{1}^{\prime} \sin \phi_{1} \cdot \rho_{2}^{\prime} \sin \phi_{2}\right\}
\end{aligned}
$$

The mutual inductance, $m_{w 1 w 2}$, between the two helical wires, $w_{1}$ and $w_{2}$, is found by multiplying this expression for $d I_{1} d I_{2}$ by $m$ and integrating over both circular sections of the wire, an operation which is equivalent to multiplying by 1 when applied to any terms of $m$ except the finite part $m_{\theta}$ since the operation only alters the subject by a secondorder fraction of itself.

Now, by eq 13 , replacing the $x$ by $x^{\prime}$

$$
\begin{aligned}
\iint d I_{1} \iint d I_{2} m_{\theta}=\iint d I_{1} \iint d I_{2} \omega_{\theta}\left(x_{e_{2}}^{\prime}-x_{i_{1}}^{\prime}\right)-\iint d I_{1} \iint d I_{2} \omega_{\theta}\left(x_{\theta_{s}}^{\prime}-x_{\theta_{1}}^{\prime}\right) \\
+\iint d I_{1} \iint d I_{2} \omega_{\theta}\left(x_{i_{2}}^{\prime}-x_{e_{1}}^{\prime}\right)-\iint d I_{1} \iint d I_{2} \omega_{\theta}\left(x_{i_{2}}^{\prime}-x_{i_{1}}^{\prime}\right) .
\end{aligned}
$$

Also

$$
\begin{aligned}
\omega_{0}\left(x_{e_{2}}^{\prime}-x_{i_{1}}^{\prime}\right) & =\omega_{\theta}\left(x_{e_{3}}^{\prime}-x_{i_{i}}^{\prime}, r_{1}^{\prime}, r_{2}^{\prime}\right) \\
& =\omega_{\theta}\left(x_{e_{2}}-x_{i_{1}}+\rho_{2}^{\prime} \cos \phi_{2}-\rho_{1}^{\prime} \cos \phi_{1}, r_{1}+\rho_{1} \sin \phi_{1}, r_{2}+\rho_{2} \sin \phi_{2}\right),
\end{aligned}
$$

where $\mathrm{x}_{e_{3}}-x_{i_{1}}, r_{1}$, and $r_{2}$ refer to the central filaments of the wires. The expansion of this by Taylor's theorem for a function of three variables may be written symbolically (to the second order in $\rho_{1}^{\prime}$ and $\left.\rho_{2}^{\prime}\right)$

$$
\begin{gathered}
\omega_{\theta}\left(x_{\theta_{3}}^{\prime}-x_{i}^{\prime}\right)=\left\{1+\left[\left(\rho_{2}^{\prime} \cos \phi_{2}-\rho_{1}^{\prime} \cos \phi_{1}\right) D_{x}+\rho_{1}^{\prime} \sin \phi_{1} D_{r_{1}}+\rho_{2}^{\prime} \sin \phi_{2} D_{r_{2}}\right]\right. \\
\left.+\frac{1}{2}\left[\left(\rho_{2}^{\prime} \cos \phi_{2}-\rho_{1}^{\prime} \cos \phi_{1}\right) D_{x}+\rho_{1}^{\prime} \sin \phi_{1} D_{r_{1}}+\rho_{2}^{\prime} \sin \phi D_{r_{2}}\right]^{2}\right\} \omega_{\theta}\left(x, r_{1} r_{2}\right),
\end{gathered}
$$

where $x=x_{e_{2}}-x_{i_{1}}$. 
Multiplying eq 63 by eq 66 and integrating gives

$$
\begin{aligned}
& \iint d I_{1} \iint d I_{2} \omega_{\theta}\left(x_{\theta_{1}}^{\prime}-x_{i_{1}}^{\prime}\right) \\
& \quad=\left\{1+\frac{\rho_{1}^{2}}{8}\left(D_{x}^{2}+D_{r_{1}}^{2}+2 A_{1} D_{r_{1}}\right)+\frac{\rho_{2}^{2}}{8}\left(D_{x}^{2}+D_{r_{2}}^{2}+2 A_{2} D_{r_{2}}\right)\right\} \omega_{\theta}\left(x, r_{1} r_{2}\right),
\end{aligned}
$$

where $x=x_{e_{2}}-x_{i_{1}}=x=$ the distance between end plane of the central filament of wire $w_{2}$ and the initial plane of the central filament of wires $w_{1}$. On making use of the partial differential 36 , this becomes so that

$$
\begin{gathered}
\iint d I_{1} \iint d I_{2} \omega_{\theta}\left(x_{e_{2}}^{\prime}-x_{i_{1}}^{\prime}\right)=\omega_{\theta}\left(x_{e 2}-x_{i 1}\right) \\
+\frac{1}{8}\left[\rho_{1}^{2}\left(\frac{1}{r_{1}}+2 A_{1}\right) D_{r_{1}}+\rho_{2}^{2}\left(\frac{1}{r_{2}}+2 A_{2}\right) D_{r_{2}}\right] \omega_{\theta}+\frac{\rho_{1}^{2}+\rho_{2}^{2}}{8} \frac{\sqrt{r_{1} r_{2}}}{\rho_{1} \rho_{2}} \phi_{1}\left(k_{0}\right) .
\end{gathered}
$$

The expansion of the three remaining integrals of eq 64 is the same as this with the appropriate $x$-arguments.

The result is that, to the second order, the mutual inductance of the two wires is given by

$$
m_{w 1 w 2}=m+\frac{1}{8}\left[\rho_{1}^{2}\left(\frac{1}{r_{1}}+2 A_{1}\right) D_{r_{1}}+\rho_{2}^{2}\left(\frac{1}{r_{2}}+2 A_{2}\right) D_{r_{2}}\right] m_{\theta},
$$

where $h_{1}$ and $h_{2}$ are their central helical filaments, with radii $r_{1}$ and $r_{2}$. This may be written

$$
m_{w 1 w 2}=m_{\theta}\left(\bar{r}_{1}, \bar{r}_{2}\right)+m_{x}+m_{a},
$$

where

$$
\bar{r}_{1}=r_{1}+\frac{\rho_{1}^{2}}{8}\left(\begin{array}{c}
1 \\
r_{1}
\end{array}+2 A_{1}\right) \text { and } \overline{r_{2}}=r+\frac{\rho_{2}^{2}}{8}\left(1+2 A_{2}\right),
$$

where all the $m$ 's except the principal one refer to the central helices of the two wires, and every $m$ is expressed in terms of its $w$-function, as in eq 13, with corresponding subscripts.

The sole effect of giving the wires a radius is to increase the effective radii of their current sheets from $r$ to $\bar{r}$ (to the second order), the length of the sheets being unaltered. The latter fact is explained in part by the particular manner in which their lengths have been defined, and in part by the fact that the wire sections are circular, so that the terms which would represent an increase in effective lengthbeing proportional to $D_{x}^{2} \omega_{\theta}$-have entered the result together with $D_{r}^{2} \omega_{\theta}$ and their combined effects have been expressed in terms of $D_{r_{1} \omega_{\theta}}$ and $D_{r_{2}} \omega_{\theta}$ by means of the partial differential eq 36 .

For distributions of current in both wires whose $r$-derivative vanishes at the central filament, the constants $A_{1}$ and $A_{2}$ are both zero. 
The uniform current distribution is an example of this class. A correction equal to the above but opposite in sign is obtained for distributions in which $A_{1}=-1 / r_{1}$, and $A_{2}=-1 / r_{2}$, an example of this class being the "natural" distribution $\left(B_{1}=1 / r_{1}^{2}\right)$, where the current density varies inversely as the linear length $\sqrt{r^{2}+\rho^{2}}$ of the helical current filaments. A distribution for which $A_{1}=-1 / 2 r_{1}, A_{2}=-1 / 2 r_{2}$ would have no correction of this order of magnitude.

Whatever assumption be made as to the current distribution, it will still be necessary to evaluate $D_{r_{1}} m$ and $D_{r_{2}} m$ roughly in order to estimate the effects of errors in determination of the mean radii, $r_{1}$ and $r_{2}$.

At present we are ignorant of the current distribution in such wires, so that for simplicity it would seem better to ignore altogether this correction for finite thickness of the wires. The assumption usually made of uniform current density carries with it a spurious precision.

We can, however, allow for future increase of knowledge as to current distribution, with no less simplicity of treatment, by defining the helix which is equivalent to a helical wire as one having the same pitch and end planes as its central filament, but a cylindrical radius

$$
r=a+\frac{\rho^{2}}{8}\left(\frac{1}{a}+2 A\right)
$$

where $a$ is the mean cylindrical radius of the helical wire and $\rho$ is the wire-radius - the interpretation of the constant $A$ being the value at the central filament of the $r$-derivative of the current density, which was assumed to be a function of $r$ only and in the direction of the helical filament.

As far as we are concerned here, this disposes of the difference between helices and helical wires. All the formulas in the preceding sections regarding mutual inductance of two helices and the force between them are valid for the helical wires to which they are equivalent.

\section{APPLICATION TO THE CURRENT BALANCE USED IN THE NATIONAL PHYSICAL LABORATORY}

If the $x$ axis be taken vertically upward, the lower (or suspended) helix, called No. 1 , has a mean radius $r_{1}=10 \mathrm{~cm}$ and length $l_{1}=15.2$ $\mathrm{cm}$. The upper helix has radius $r_{2}=16 \mathrm{~cm}$ and length $l_{2}=11.0 \mathrm{~cm}$. Both helices have the same pitch, $2 \pi p_{1}=2 \pi p_{2}=0.2 \mathrm{~cm}$ and an integral number of turns, $N_{1}=76$ and $N_{2}=55$ turns. Hence, every $\theta_{x}=\alpha=$ $\theta_{i_{2}}-\theta_{i_{1}}$ the azimuth of the helices. The upper end of No. 1 projects into No. 2, so that the third $x$-difference of eq 11 is negative.

$$
\begin{aligned}
& x_{1} \equiv x_{e_{2}}-x_{i_{1}}=+20.1 \mathrm{~cm} \rightarrow k_{1}{ }^{2}=0.5926 \\
& x_{2} \equiv x_{e_{2}}-x_{e_{1}}=+4.9 \mathrm{~cm} \rightarrow k_{2}{ }^{2}=0.9143 \\
& x_{3} \equiv x_{i_{2}}-x_{e_{1}}=-6.1 \mathrm{~cm} \rightarrow k_{3}{ }^{2}=0.8974 \\
& x_{4} \equiv x_{i_{2}}-x_{i_{1}}=+9.1 \mathrm{~cm} \rightarrow k_{4}{ }^{2}=0.8434
\end{aligned}
$$

The force, $f$, acting on the suspended helix when both carry unit current is

$$
f=f_{\theta}+\bar{f}_{x}(\alpha)+f_{a}(\alpha),
$$


where $f_{\theta}$ is the principal part due to angular components of current in the helices and by eq $13^{\prime}$.

$$
f_{\theta}=-\omega_{\theta}^{\prime}\left(x_{1}\right)+\omega_{\theta}^{\prime}\left(x_{2}\right)-\omega_{\theta}^{\prime}\left(x_{3}\right)+\omega_{\theta}^{\prime}\left(x_{4}\right),
$$

where the $\omega \theta^{\prime}(x)$ are given by eq 39 . The small part $\bar{f}_{x}(\alpha)$ denotes the residue due to $x$-components of current in helices and lead wires together and is given by

$$
\bar{f}_{x}(\alpha)=-\bar{\omega}_{x}^{\prime}\left(x_{1}, \alpha\right)+\bar{\omega}_{x}^{\prime}\left(x_{2}, \alpha\right)-\bar{\omega}_{x}^{\prime}\left(x_{3}, \alpha\right)+\bar{\omega}_{x}^{\prime}\left(x_{4}, \alpha\right),
$$

where the $\bar{\omega}_{x}^{\prime}(x, \alpha)$ are given by eq $46^{\prime}$.

The last part, $f_{o}(\alpha)$, is given similarly by

$$
f_{a}(\alpha)=-\omega_{a}^{\prime}\left(x_{1}, \alpha\right)+\omega_{a}^{\prime}\left(x_{2}, \alpha\right)-\omega_{a}^{\prime}\left(x_{5}, \alpha\right)+\omega_{a}^{\prime}\left(x_{4}, \alpha\right),
$$

where the $\omega_{a}^{\prime}(x, \alpha)$ may be computed by eq $49^{\prime}$ or $57^{\prime}$. Both $\bar{\omega}_{x}^{\prime}(x, \alpha)$ and $\omega_{a}^{\prime}(x, \alpha)$ are even periodic functions of $\alpha$, with period $2 \pi$, so the same is true of $\vec{f}_{x}(\alpha)$ and $f_{a}(\alpha)$.

In the actual coils, however, there are two helices on each cylinder differing in azimuth by $\pi$, so that when each carries unit current the force on the suspended coil is $F$, where

$$
F=F_{\theta}+\bar{F}_{x}(\alpha)+F_{a}(\alpha),
$$

where

$$
\left.\begin{array}{rl}
F_{\theta} & =4 f_{\theta} \\
\bar{F}_{x}(\alpha) & =2\left[\vec{f}_{x}(\alpha)+\bar{f}_{x}(\alpha+\pi)\right] \\
F_{a}(\alpha) & =2\left[f_{a}(\alpha)+f_{a}(\alpha+\pi)\right]
\end{array}\right\}
$$

This makes the azimuthal terms, $\bar{F}_{x}(\alpha)$ and $F_{a}(\alpha)$, each even periodic functions of $\alpha$, with period $\pi$, so that

$$
\bar{F}_{x}(\alpha)+F_{a}(\alpha)=\sum_{n=1}^{\infty} C_{2 n} \cos 2 n \alpha .
$$

P. Vigoureux of the National Physical Laboratory of England has recently reported to this Bureau that in the absence of a formula for the coefficients $C_{2 n}$ he has evaluated experimentally the first coefficient, $C_{2}$, assuming the first term to be the only one of the series of practical importance. This he did by measuring the force for three different azimuths $\alpha=3^{\circ}, 15^{\circ}$, and $85^{\circ}$.

In order to see what part of $C_{2}$ is due to axial components of current and what part is due to the azimuth terms $\omega_{a}^{\prime}$, let

$$
C_{2 n}=C_{2 n}^{x}+C_{2 n}^{a} \text {. }
$$

Reference to eq 72,76 , and $46^{\prime \prime}$ shows that the effect of axial currents is given by

$$
C_{2 n}^{x}=\frac{1}{\sqrt{r_{1} r_{2}}} \cdot \frac{1}{n}\left(\frac{r_{1}}{r_{2}}\right)^{2 n}\left\{-x_{1} k_{1}+x_{2} k_{2}-x_{3} k_{3}+x_{4} k_{4}\right\}
$$


and eq $49^{\prime}$ shows that

$$
\begin{aligned}
C_{2 n}^{a}=\frac{1}{\sqrt{r_{1} r_{2}}} & \frac{1}{4 n^{2}}\left\{-x_{1} k_{1}^{3}\left[\phi_{2 n+1}^{\prime}\left(k_{1}\right)+\phi_{2 n-1}^{\prime}\left(k_{1}\right)\right]+x_{2} k_{2}^{3}\left[\phi_{2 n+1}\left(k_{2}\right)+\phi_{2 n-1}\left(k_{2}\right)\right]\right. \\
& \left.-x_{3} k_{3}^{3}\left[\phi_{2 n+1}^{\prime}\left(k_{3}\right)+\phi_{2 n-1}^{\prime}\left(k_{3}\right)\right]+x_{4} k_{4}^{3}\left[\phi_{2 n+1}\left(k_{4}\right)+\phi_{2 n-1}\left(k_{4}\right)\right]\right\}
\end{aligned}
$$

Placing $n=1$

$$
\begin{array}{r}
C_{2}^{x}=\frac{1}{\sqrt{r_{1}} r_{2}}\left(\frac{r_{1}}{r_{2}}\right)^{2}\left[-x_{1} k_{1}+x_{2} k_{2}-x_{3} k_{3}+x_{4} k_{4}\right]=+0.103 \text { dynes } \\
\left.C_{2}^{a}=\frac{1}{\sqrt{r_{1} r_{2}}}\right\}-x_{1} k_{1}^{3}\left[\frac{\phi_{1}^{\prime}\left(k_{1}\right)+\phi_{3}^{\prime}\left(k_{1}\right)}{4}\right]+x_{2} k_{2}^{3}\left[\frac{\phi_{1}^{\prime}\left(k_{2}\right)+\phi_{3}^{\prime}\left(k_{2}\right)}{4}\right] \\
\left.-x_{3} k_{3}^{3}\left[\frac{\phi_{1}^{\prime}\left(k_{3}\right)+\phi_{3}^{\prime}\left(k_{3}\right)}{4}\right]+x_{4} k_{4}^{3}\left[\frac{\phi_{1}^{\prime}\left(k_{4}\right)+\phi_{3}^{\prime}\left(k_{4}\right)}{4}\right]\right\}
\end{array}
$$

From the recurrence relations 20 and 21 , it is found that

$$
\begin{aligned}
k^{3}\left[\frac{\phi_{1}^{\prime}(k)+\phi_{3}^{\prime}(k)}{4}\right] & =\frac{1}{\pi^{2}}\left\{\left[\frac{1}{2}+\frac{8 k^{\prime 2}}{3 k^{4}}\right] \pi^{2} k^{3} \phi_{1}^{\prime}(k)-\left(\frac{2}{k^{2}}-1\right) 4 \pi^{2} \phi_{1}(k)\right\} \\
& =\frac{1}{\pi^{2}}\left\{\left[\frac{1}{2}+\frac{8 k^{\prime 2}}{3 k^{4}}\right] Y_{2}(k)-\left(\frac{2}{k^{2}}-1\right) Y_{1}(k)\right\} \equiv \frac{1}{\pi^{2}} Y(k),
\end{aligned}
$$

where $Y_{1}$ and $Y_{2}$ are the functions defined in eq 23, which may be taken from tables.

$$
\begin{aligned}
Y_{1}(k) & =2.086, \quad 9.100, \quad 8.165, \text { and } 6.072 \text { for } k_{1}, k_{2}, k_{3}, \text { and } k_{4} \\
Y_{2}(k) & =1.490, \quad 25.663, \quad 20.049, \text { and } 10.796 \\
Y(k) & =0.401, \quad 9.04, \quad 6.57, \text { and } 3.41 \\
C_{2}^{a} & =\frac{1}{\pi^{2} \sqrt{r_{1} r_{2}}}\left\{-x_{1} Y\left(k_{1}\right)+x_{2} Y\left(k_{2}\right)-x_{3} Y\left(k_{3}\right)+x_{4} Y\left(k_{4}\right)\right\}=0.86 \text { dyne. }
\end{aligned}
$$

\section{Hence}

$C_{2}^{a}+C_{2}^{x}=0.86+0.10=0.96$ dyne, which differs from the observed value 1 dyne by less than the experimental error.

The force $F_{\theta}=94,527.22$ dynes, so that

$$
F=94,527.22+0.96 \cos 2 \alpha
$$

For azimuth $\alpha=0$ or $\pi$, this azimuthal term amounts to 10 parts in a million.

\section{SUMMARY}

The self inductance between two coaxial helices and the force between them are given in terms of four $\omega$-functions, and their $x$ derivatives, respectively, as in eq 13 and $13^{\prime}$, the four $x$-distances being defined in eq 11 with their respective azimuthal differences $\theta_{x}$. Resolving these functions into three components, $\omega(x)=\omega_{\theta}(x)+\omega_{x}(x)$ $+\omega_{a}\left(x, \theta_{x}\right)$, the principal part corresponds to the mutual inductance 
between the two associated current sheets, with angular components of current, and $\omega_{x}(x)$ with axial components. The last term, $\omega_{a}\left(x, \theta_{x}\right)$, is like $\omega_{x}(x)$, a second-order correction term applied to the finite term, $\omega_{\theta}(x)$, where the ratio of pitch to cylindrical radius is considered a first-order infinitesimal.

The principal term, $\omega_{\theta}(x)$, is given by eq 37 and $\omega_{x}(x)$ by eq 39 . The first is equivalent to Jones' formula for current sheets. The terms $\omega_{x}(x)$ and $\omega_{x}^{\prime}(x)$ are given by eq 42 and 43 .

The azimuthal terms, $\omega_{a}\left(x, \theta_{x}\right)$ and $\omega_{a}^{\prime}\left(x, \theta_{x}\right)$, are given by the series eq 49 and $49^{\prime}$ and in finite terms by eq 57 and $57^{\prime}$.

These formulas are not restricted to the case where the helices have an integral number of turns but are quite general. They need not have the same pitch. For the case most common in practice where the numbers of turns are integers and the lead wires are straight lines parallel and close to the generator of the cylindrical current sheet, the total effect of all axial components of current in helices and lead wires together is represented by a function $\bar{\omega}_{x}(x)$ which is given by eq 46 , and its $x$-derivative by eq $46^{\prime}$.

It is shown in section VII that the azimuthal variation of the force computed by these equations agrees (within experimental errors) with that observed at the National Physical Laboratory.

The correction terms given in section VI take account of the finite diameter of helical wires. They require a knowledge of the current distribution in the wires.

These formulas are valid to the second order, inclusive, provided no parts of the two helices lie very close to each other, that is, at distances of the order of magnitude of the pitch of the windings.

Washington, December 21, 1938. 